\title{
elF2a phosphorylation is pathognomonic for immunogenic cell death
}

\author{
Lucillia Bezu ${ }^{1,2,3,4,5,6}$ - Allan Sauvat ${ }^{2,3,4,5,6}$ - Juliette Humeau ${ }^{1,2,3,4,5,6}$ - Lígia C. Gomes-da-Silva 2,3,4,5,6,7 •

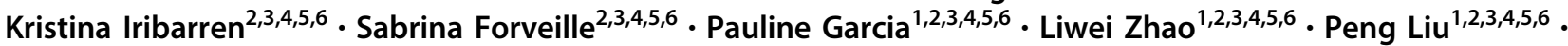 \\ Laurence Zitvogel ${ }^{1,8,9} \cdot$ Laura Senovilla ${ }^{2,3,4,5,6} \cdot$ Oliver Kepp ${ }^{2,3,4,5,6} \cdot$ Guido Kroemer $^{2,3,4,5,6,10,11}$
}

Received: 4 May 2017 / Revised: 13 November 2017 / Accepted: 15 November 2017 / Published online: 22 January 2018

(c) ADMC Associazione Differenziamento e Morte Cellulare 2018

\begin{abstract}
The phosphorylation of eIF2 $\alpha$ is essential for the endoplasmic reticulum (ER) stress response, the formation of stress granules, as well as macroautophagy. Several successful anticancer chemotherapeutics have the property to induce immunogenic cell death (ICD), thereby causing anticancer immune responses. ICD is accompanied by the translocation of calreticulin (CALR) from the ER lumen to the plasma membrane, which facilitates the transfer of tumor-associated antigens to dendritic cells. Here we systematically investigated the capacity of anticancer chemotherapeutics to induce signs of ER stress. ICD inducers including anthracyclines and agents that provoke tetraploidization were highly efficient in enhancing the phosphorylation of eIF2 $\alpha$, yet failed to stimulate other signs of ER stress including the transcriptional activation of activating transcription factor 4 (ATF4), the alternative splicing of X-box binding protein 1 (XBP1s) mRNA and the proteolytic cleavage of activating transcription factor 6 (ATF6) both in vitro and in cancers established in mice. Systematic analyses of clinically used anticancer chemotherapeutics revealed that only eIF2 $\alpha$ phosphorylation, but none of the other signs of ER stress, correlated with CALR exposure. eIF2 $\alpha$ phosphorylation induced by mitoxantrone, a prototype ICD-inducing anthracyline, was mediated by eIF $2 \alpha$ kinase-3 (EIF2AK3). Machine-learning approaches were used to determine the physicochemical properties of drugs that induce ICD, revealing that the sole ER stress response relevant to the algorithm is eIF2 $\alpha$ phosphorylation with its downstream consequences CALR exposure, stress granule formation and autophagy induction. Importantly, this approach could reduce the complexity of compound libraries to identify ICD inducers based on their physicochemical and structural characteristics. In summary, it appears that eIF $2 \alpha$ phosphorylation constitutes a pathognomonic characteristic of ICD.
\end{abstract}

\section{Introduction}

The unprecedented surge of immunotherapies has demonstrated to which extent the relationship between the immune system and malignant cells determines the biology of cancer $[1,2]$. Although immune checkpoint blockade (ICB) targeting CTLA-4, PD-1 or PD-L1 has yielded spectacular

Edited by S. Fulda

Electronic supplementary material The online version of this article (https://doi.org/10.1038/s41418-017-0044-9) contains supplementary material, which is available to authorized users.

\section{Oliver Kepp}

captain.olsen@gmail.com

$\triangle$ Guido Kroemer

kroemer@orange.fr

Extended author information available on the last page of the article therapeutic responses in many cancers, it currently appears that none of these immunotherapies will provide definitive cure of frequent tumor types [3, 4]. Moreover, clinical trials have demonstrated that ICB is largely inefficient against most mammary and colorectal cancer subtypes, which, in contrast to other neoplasias, are manageable with conventional therapies $[5,6]$.

Several chemotherapeutic agents that have been particularly successful, including anthracyclines, oxaliplatin and microtubular inhibitors (such as taxanes and vinca-alkaloids) as well as radiotherapy, stimulate anticancer immune responses via a particular mechanism, i.e., the induction of immunogenic cell death (ICD) [7-9]. ICD constitutes a variety of (mostly apoptotic and sometimes necroptotic) cell death that is preceded by two major premortem stress responses, i.e., autophagy and endoplasmic reticulum (ER) stress $[10,11]$. Autophagy is required for the lysosomal secretion of ATP, which, when present in the extracellular 
space, acts as a potent chemotactic factor that attracts myeloid cells including dendritic cell (DC) precursors into the tumor bed [11-15]. ER stress leads to the translocation of calreticulin (CALR), which is the most abundant protein contained in the ER lumen, to the plasma membrane, where it acts as an 'eat-me' signal to facilitate the uptake of tumorassociated antigens by DC $[10,16]$. DC then cross-present tumor antigens to cytotoxic T cells, setting of an anticancer immune response [13, 17].

Experiments in preclinical models have demonstrated that chemotherapy with ICD inducers as well as radiotherapy is far more efficient in causing tumor growth reduction when an anticancer immune response is launched $[18,19]$. Thus, immune defects largely abolish the efficacy of chemotherapy [20], while ICB can enhance the anticancer effects of ICD inducers in mouse models [21]. Similarly, patients with colorectal or mammary carcinoma fail to respond to chemotherapy if they lack cytotoxic $\mathrm{T}$ lymphocytes in the tumor $[16,22,23]$ and if they bear genetically determined defects that hamper the recognition of ICD [17, 24, 25].

It is important to note that most chemotherapeutic agents are unable to induce ICD, meaning that they kill cancer cells in a way that is neutral (or potentially tolerogenic) with respect to immune recognition. For example, cisplatin, which is the most widely used cytotoxicant, is unable to elicit ICD, while oxaliplatin is highly efficient in doing so $[20,26]$. This led to the design of screening programs in which the hallmarks of ICD (ATP release, CALR exposure, as well as nuclear release of HMGB1) were determined in cultured human cancer cells to identify drugs with immunostimulatory properties [27-29]. Moreover, the detection of ICD-related features on human cancer samples has yielded prognostic biomarkers. For example, the loss of CALR expression by malignant cells correlates with weak anticancer immune responses and poor prognosis in non-small cell lung cancer, as well as in acute myeloid leukemia $[30,31]$. In this context, it turned out that CALR expression correlates with one particular sign of ER stress, which is the phosphorylation of eukaryotic initiation factor $2 \alpha(\operatorname{eIF} 2 \alpha)$ on serine $51[30,31]$.

The phosphorylation of eIF $2 \alpha$ is catalyzed by at least four distinct specific kinases (the eIF $2 \alpha$ kinases, EIF2AK1-4) and regulated by at least two distinct phosphatases, one of which is protein phosphatase 1 (PP1) with its cofactor DNA damage-inducible 34 (GADD34) [32, 33]. eIF $2 \alpha$ phosphorylation is the hallmark of the 'integrated stress response' (ISR) that occurs in the context of ER stress [34-36]. ER stress is under the control of three pathways governed by three stress sensors: protein kinase RNA-like ER kinase (PERK, also called EIF2AK3), inositol-requiring protein $1 \alpha(\operatorname{IRE} 1 \alpha)$ and activating transcription factor 6
(ATF6) $[37,38]$. PERK phosphorylates eIF2 $\alpha$ (P-eIF2 $\alpha$ ), thereby stopping CAP-dependent mRNA translation and favoring the translation of the mRNA coding for activating transcription factor 4 (ATF4). ATF4 protein then acts as a transcription factor to induce DNA damage-inducible transcript 3 (DDIT3, best known as C/EBP homologous protein, $\mathrm{CHOP}$ ). $\mathrm{CHOP}$ in turn facilitates the transcription of GADD34, which realizes a negative feedback on P-eIF2 $\alpha$ to maintain ER homeostasis. Active IRE1 possesses in its cytoplasmic domain an endoribonuclease function allowing it to excise an intron of 26 nucleotides from the mRNA of the transcription factor $\mathrm{X}$ box-binding protein 1 (XBP1). Spliced XBP1 (XBP1s) translocates to the nucleus and induces the transcription of target genes involved in ERassociated degradation (ERAD). Finally, ATF6 is cleaved specifically by two proteases in the Golgi apparatus. The cleaved form (p50 ATF6) translocates to the nucleus where it also controls genes encoding ERAD components. Importantly, eIF $2 \alpha$ phosphorylation is not only involved in the ER stress response (also termed unfolded protein response') that culminates in CALR exposure [39], but is also essential for the stimulation of stress granules, which are stalled translation pre-initiation complexes [40] and for the induction of macroautophagy [41, 42].

Altogether it appears that the ISR, ER stress response and ICD share several common features. Driven by this premise, we decided to investigate all signs of stress occurring in the context of the ISR and ER stress in the context of ICD. Here, we reveal the surprising finding that among the three arms of the ER stress response, only eIF2 $\alpha$ phosphorylation is relevant to ICD both in vitro and in vivo.

\section{Results}

\section{Anthracyclines and oxaliplatin induce an incomplete ER stress response}

ICD inducing chemotherapeutics such as oxaliplatin and the anthracyclines mitoxantrone and doxorubicin stimulate the phosphorylation of eIF $2 \alpha$ at serine 51 as determined by immunoblot detection of phosphorylated eIF $2 \alpha$ using a phospho-neoepitope-specific antibody (Fig. 1a, b). This effect of doxorubicin, mitoxantrone and oxaliplatin resembled to that of well-known ER stress inducers such as tunicamycin (which inhibits $\mathrm{N}$-linked glycosylation of protein and hence causes an overload of proteins in the ER and the Golgi) [43] and thapsigargin (which noncompetitively inhibits the sarco/endoplasmic reticulum $\mathrm{Ca}^{2+}$ ATPase, SERCA, thereby reducing ER $\mathrm{Ca}^{2+}$ concentrations) [44]. In sharp contrast, doxorubicin, mitoxantrone and oxaliplatin failed to stimulate the expression of 
Fig. 1 Anthracyclines induce the phosphorylation of eIF $2 \alpha$ yet fail to induce other markers of ER stressa-h Cells were treated with mitoxantrone (MTX, 2 $\mu \mathrm{M})$, doxorubicin (DOXO, 2 $\mu \mathrm{M})$, oxaliplatin (OXA, 500 $\mu \mathrm{M})$, tunicamycin (TM, $3 \mu \mathrm{M})$, thapsigargin (TG, $3 \mu \mathrm{M})$, cisplatin (CDDP, $150 \mu \mathrm{M}$ ), resveratrol (RESV, $50 \mu \mathrm{M})$, spermidine (SPD, $50 \mu \mathrm{M})$ and rapamycin (RAPA, $10 \mu \mathrm{M}$ ). a-h Human osteosarcoma U2OS cells were treated for $6 \mathrm{~h}$ $(\mathbf{a}-\mathbf{b})$ or $12 \mathrm{~h}(\mathbf{c}-\mathbf{f})$, harvested, and proteins were separated by SDS-polyacrylamid gel electrophoreses and following detected by immunoblot. Representative immunoblots $(\mathbf{a}, \mathbf{c}, \mathbf{e})$ and densitometry data $\mathbf{b}$, d, $\mathbf{f}$ are depicted. Densitometry data are represented as mean value \pm SD of three independent experiments ( $n=4$ for b). e, $\mathbf{f}$ U2OS cells stably expressing $\mathrm{XBP} 1 \triangle \mathrm{DBD}$-venus were treated for $12 \mathrm{~h}$ and stained with a mouse anti-XBP1s antibody. g-h U2OS were treated for $6 \mathrm{~h}$ with the indicated drugs then the cells were harvested and total RNA was isolated. Reverse transcriptase polymerase chain reaction (RT PCR) was performed with primers specific for human XBP1s. Then the PCR products were separated by gel electrophoresis.

Representative images (g) and densitometry data (h) shown as mean value \pm SD of three independent experiments are depicted. Samples were compared using Student's $t$ test $(* p<0.05, * * p<0.01$, $* * * p<0.001)$
A

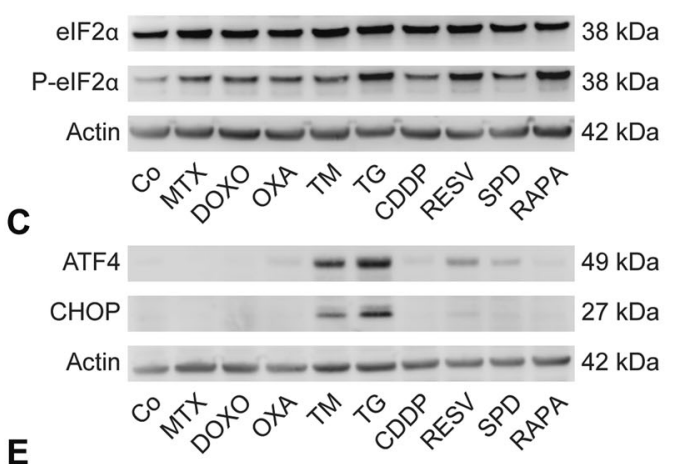

E

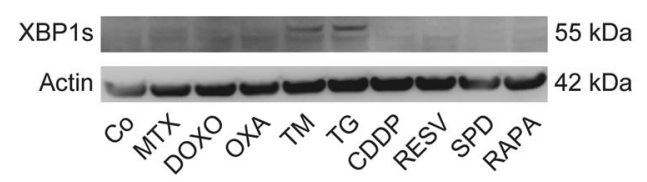

G

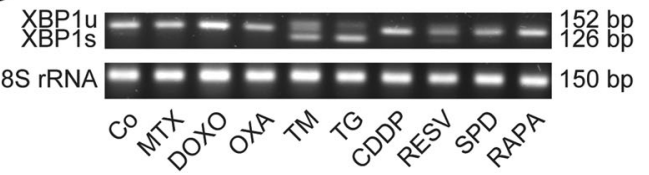

B

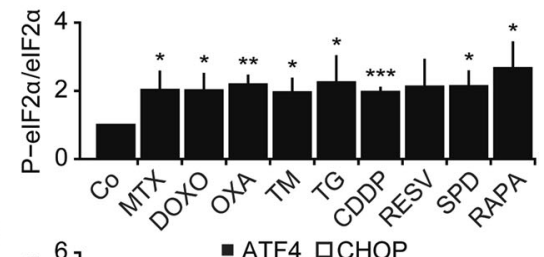

D

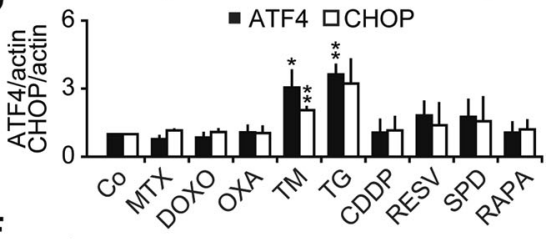

F

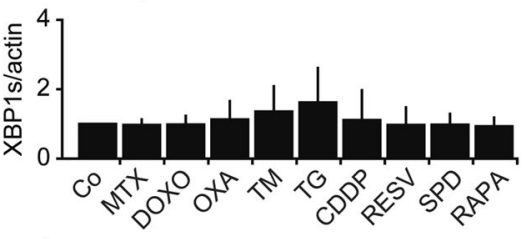

$\mathrm{H}$

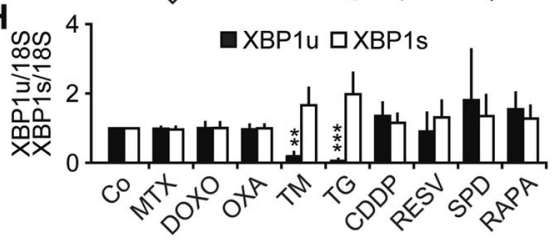

two transcription factors that theoretically should be activated downstream of eIF $2 \alpha$ phosphorylation, ATF4 and CHOP, as determined by immunoblots of whole-cell extracts (Fig. 1c, d). Moreover, doxorubicin, mitoxantrone and oxaliplatin were unable to cause the IRE1-mediated splicing of XBP1, as assessed by immunoblot or RT-PCR detection of the XBP1s isoform (Fig. 1e-h). Of note, several autophagy inducers (resveratrol, spermidine, rapamycin) that operate through distinct mechanisms elicited a similar response pattern, meaning that they stimulated eIF $2 \alpha$ phosphorylation, which is known to be required for autophagy initiation yet failed to activate ATF4, CHOP and XBP1 splicing (Fig. 1a-h).
The aforementioned results were reproduced when the hallmarks of ER stress were evaluated by alternative cellbased methods, namely by immunofluorescence detection of eIF $2 \alpha$ phosphorylation or the use of appropriate biosensor cell lines that allow for the detection of the transcriptional activation of GFP placed under the control of the ATF4 promoter, the redistribution of a GFP-ATF6 fusion protein to the Golgi and the nucleus, or the expression of an $\mathrm{XBP} 1 \Delta \mathrm{DBD}$-venus fusion protein that is only in-frame for venus (a variant of GFP), when XBP1 has been spliced by IRE1 (Fig. 2). MTX induced eIF2 $\alpha$ phosphorylation (Fig. 2a, b), as well as two phenomena known to depend on this phosphorylation event, namely CALR redistribution to 

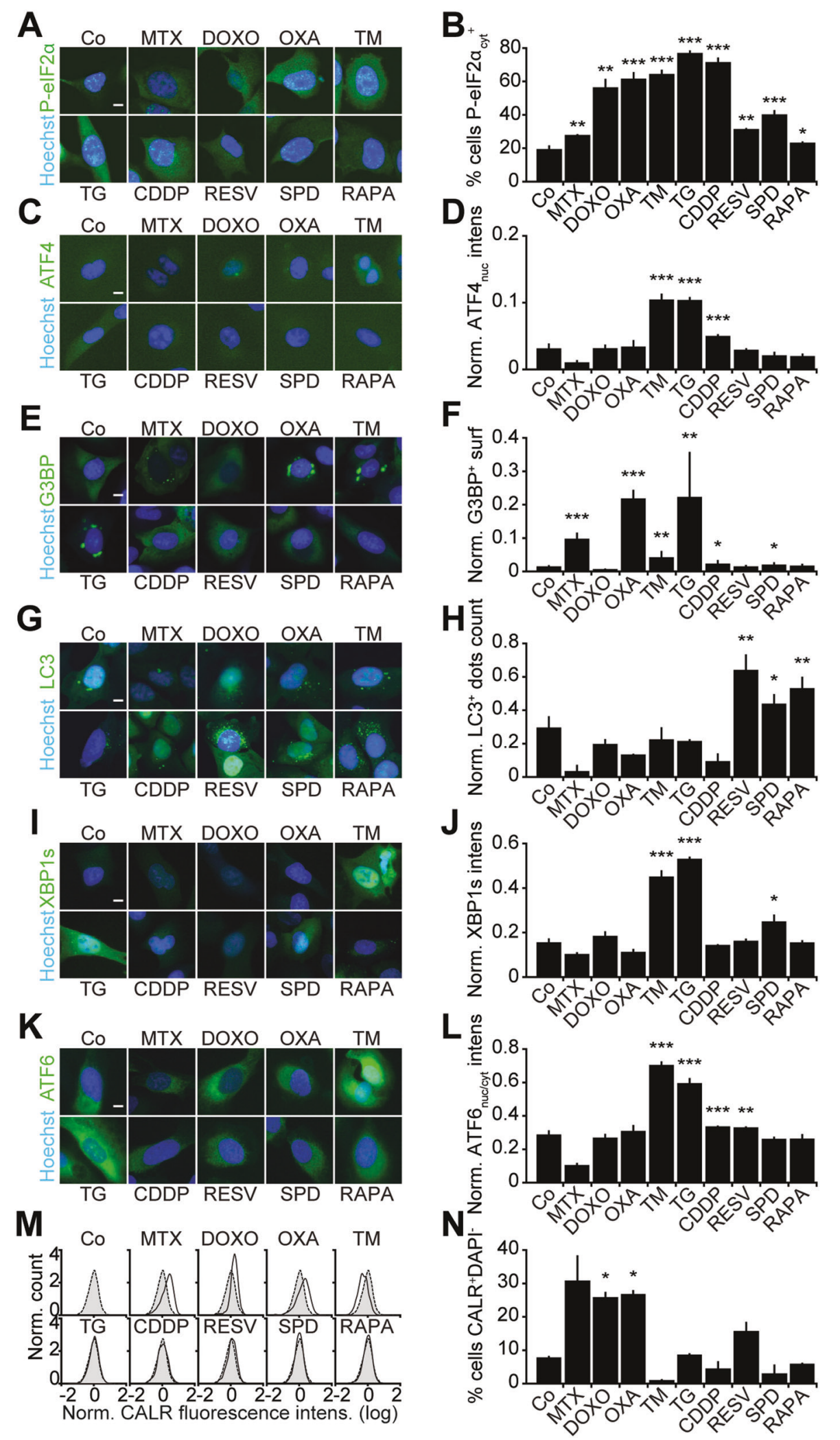
Fig. 2 Differential stress patterns evoked by immunogenic cell deathinducing and autophagy-inducing drugsHuman osteosarcoma U2OS cells stably expressing pSMALB-ATF4.5rep (that detects translational regulation via alternative usage of upstream open reading frames in the ATF4 mRNA), GFP-ATF6 (for monitoring ATF6 translocation from ER to sites of the Golgi and subsequently to the nucleus), $\mathrm{XBP} 1 \triangle \mathrm{DBD}$-venus (for monitoring venus expression upon alternative splicing of XBP1 mRNA), GFP-LC3 (for monitoring GFP-LC3 aggregation in the membranes of forming autophagosomes), G3BPGFP (for monitoring the G3BP-GFP aggregation in stress granules upon stalled translation) or parental U2OS cells were treated with the indicated agents (mitoxantrone (MTX, $3 \mu \mathrm{M}$ ), doxorubicin (DOXO, 3 $\mu \mathrm{M})$, oxaliplatin (OXA, $500 \mu \mathrm{M})$, tunicamycin (TM, $3 \mu \mathrm{M})$, thapsigargin (TG, $3 \mu \mathrm{M}$ ), cisplatin (CDDP, $150 \mu \mathrm{M}$ ), resveratrol (RESV, $50 \mu \mathrm{M}$ ), spermidine (SPD, $50 \mu \mathrm{M}$ ), rapamycin (RAPA, $3 \mu \mathrm{M})$ ) for 12 $\mathrm{h}, 6 \mathrm{~h}, 12 \mathrm{~h}, 8 \mathrm{~h}, 12 \mathrm{~h}$ and $6 \mathrm{~h}$, respectively (except G3BP-GFPexpressing cells that were treated with MTX and OXA for $24 \mathrm{~h}$ ). Data is depicted as representative images or representative histograms (a, c, e, g, i, k, m) (scale bar equals $10 \mu \mathrm{m}$ ) and was statistically evaluated by cytometric analysis of replicates $(\mathbf{b}, \mathbf{d}, \mathbf{f}, \mathbf{h}, \mathbf{j}, \mathbf{l}, \mathbf{n})$. $\mathbf{a}, \mathbf{b}$ PeIF $2 \alpha$ was assessed by means of immunofluorescence staining using a phosphoneoepitope-specific antibody and the percentage of cells with increased cytoplasmic signal is depicted. c, d pSMALB-ATF4.5rep nuclear translocation was measured by fluorescence microscopy and the average nuclear intensity of pSMALB-ATF4.5rep is depicted. e, $\mathbf{f}$ The formation of stress granules was measured by assessing the surface of G3BP-GFP-marked granules. g, h Autophagy was monitored by assessing the dots count of GFP-LC3-marked autophagosomes. $\mathbf{i}, \mathbf{j}$ XBP1s activation was measured by detecting the increase in venus fluorescence intensity. $\mathbf{k}, \mathbf{l}$ The activation of ATF6 was measured as an increment of the nuclear to cytoplasmic ratio of GFP fluorescence intensity. Data are represented as the mean \pm SD of quadruplicates from one representative out of three independent experiments. Calreticulin exposure was determined by immunofluorescence staining and flow cytometry and is expressed as percentage of calreticulin positive, DAPI negative $\left(\mathrm{CALR}^{+} \mathrm{DAPI}^{-}\right)$cells. Data are represented as the mean \pm SEM of triplicates from one representative out of three independent experiments $\mathbf{m}, \mathbf{n}$. Samples were compared using Student's $t$ test. $* p<0.05, * * p<0.01, * * * p<0.001$

the cell surface (measured by immunofluorescence of viable cells and cytofluorometry, Fig. $2 \mathrm{~m}, \mathrm{n}$ ), and redistribution of G3BP-GFP to stress granules (Figs. 2e, f). At this shortterm incubation, ICD and ER stress inducers were less efficient in stimulating autophagy than the positive controls resveratrol, spermidine and rapamycin (Fig. 2g, h). Importantly, anthracyclines and oxaliplatin failed to induce the activation of ATF4, ATF6 or XBP1 in conditions in which thapsigargin or tunicamycin yielded clearly positive results (Fig. 2c,d, i-1). This failure of ICD inducers to trigger ER stress pathways other than eIF2 $\alpha$ phosphorylation was observed over a wide range of drug concentrations and incubation periods (not shown).

To confirm this conclusion, which has been obtained by in vitro experimentation, in a more realistic in vivo setting, we implanted parental or biosensor-expressing U2OS osteosarcoma cells in immunodeficient $n u / n u$ mice (which lack a thymus and hence are devoid of thymus-dependent $\mathrm{T}$ lymphocytes) to generate macroscopic cancers (Fig. 3a). U2OS cancers exhibited immunofluorescence-detectable
eIF2 $\alpha$ phosphorylation after local injection of tunicamycin or after chemotherapy with MTX (Fig. 3b). In contrast, such cells failed to manifest the relocalization of the ATF6 biosensor to nuclei in response to chemotherapy with MTX, although they were able to mount an ER stress response at that level in vivo, in response to tunicamycin (Fig. 3d). Similarly, the positive control agent tunicamycin readily stimulated the activation of the ATF4 and XBP1s biosensors in vivo, while MTX failed to do so (Fig. 3c, e).

Altogether, these observations suggest that the in vitro results can be extrapolated to the in vivo setting. MTX does not stimulate any signs of ER stress but eIF $2 \alpha$ phosphorylation in vivo.

\section{Calreticulin exposure correlates with one single sign of ER stress}

Intrigued by the aforementioned results, we set out to determine the ER stress-inducing properties of National Cancer Institute (NCI)-approved drugs using a library of 75 approved anticancer agents, mostly including cytotoxic agents and a few tyrosine kinase inhibitors, in cell-based assays designed to measure eIF $2 \alpha$ phosphorylation, as well as CALR exposure, GFP-LC3 puncta, G3BP-GFP stress granules and the activation of ATF4, ATF6 and XBP1s. On an agent-per-agent basis, eIF $2 \alpha$ phosphorylation was positively and significantly $(p<0.05$, Pearson correlations) associated with CALR exposure, GFP-LC3 puncta, G3BPGFP stress granules, as well as with ATF4 and XBP1 activation, though not that of ATF6 (Fig. 4, Figure S1). Hence, some anticancer agents were able to stimulate the simultaneous activation of at least two arms of the ER stress response (the eIF2 $\alpha$ /ATF4 arm and the IRE1/XBP1 arm (Fig. 5a, Table S1). However, when a threshold was applied to data analysis to only include those agents that induce CALR exposure at a level that is equal or higher than mitoxantrone, the positive control for this parameter (Fig. 5b-e), it turned out that the level of CALR exposure correlated positively with eIF2 $\alpha$ phosphorylation (Pearson correlation coefficient $R=0.73, p<0.01$, Fig. 5b), yet failed to correlate with all other signs of ER stress that were measured, namely, expression of GFP under the control of the ATF4 promoter $(R=0.089$, Fig. $5 \mathrm{c})$, redistribution of GFP-ATF6 from the ER to the Golgi and the nucleus ( $R=-0.11$, Fig. $5 \mathrm{~d}$ ), and splicing of XBP1 to generate an in-frame version of the XBP1 $\triangle D B D$-venus fusion protein $(R=0.22$, Fig. $5 \mathrm{e})$.

Several chemotherapeutic agents like MTX and OXA were at least as efficient as the positive controls tunicamycin and thapsigargin to inhibit protein translation, as monitored by means of a biochemical assay evaluation the incorporation of a methionine analog into cellular proteins (Figure S2). However, only tunicamycin and thapsigargin 
Fig. 3 Partial split of ER stress responses in vivo induced by ICD drugsa A scheme showing the experimental procedure: parental U2OS and cells stably expressing pSMALBATF4.5rep, or XBP1 $\Delta$ DBDvenus were collected and injected in both flanks of immunodeficient $n u / n u$ mice. When tumors became palpable mitoxantrone (MTX, $5.17 \mathrm{mg} / \mathrm{kg}$ ), tunicamycin (TM, $1 \mathrm{mg} / \mathrm{kg}$ ) or DMEM was injected into the tumor. Transplanted U2OSwt tumors were treated for $6 \mathrm{~h}, \mathrm{U} 2 \mathrm{OS}$ pSMALB-ATF4.5rep and $\mathrm{XBP} 1 \triangle \mathrm{DBD}$-venus cells were treated $12 \mathrm{~h}$ and $48 \mathrm{~h}$ for each group treated with MTX. Then tumors were removed and fixed in $3.7 \%$ PFA. P-eIF $2 \alpha$ and ATF6 was assessed by immunostaining of parental U2OS cancers. b-e

Immunofluorescence, and GFP expression was determined by fluorescence microscopy. Results are expressed as means \pm SEM. Data were compared with $t$-test. ${ }^{*} p<0.05$, $* * p<0.01, * * * p<0.001$

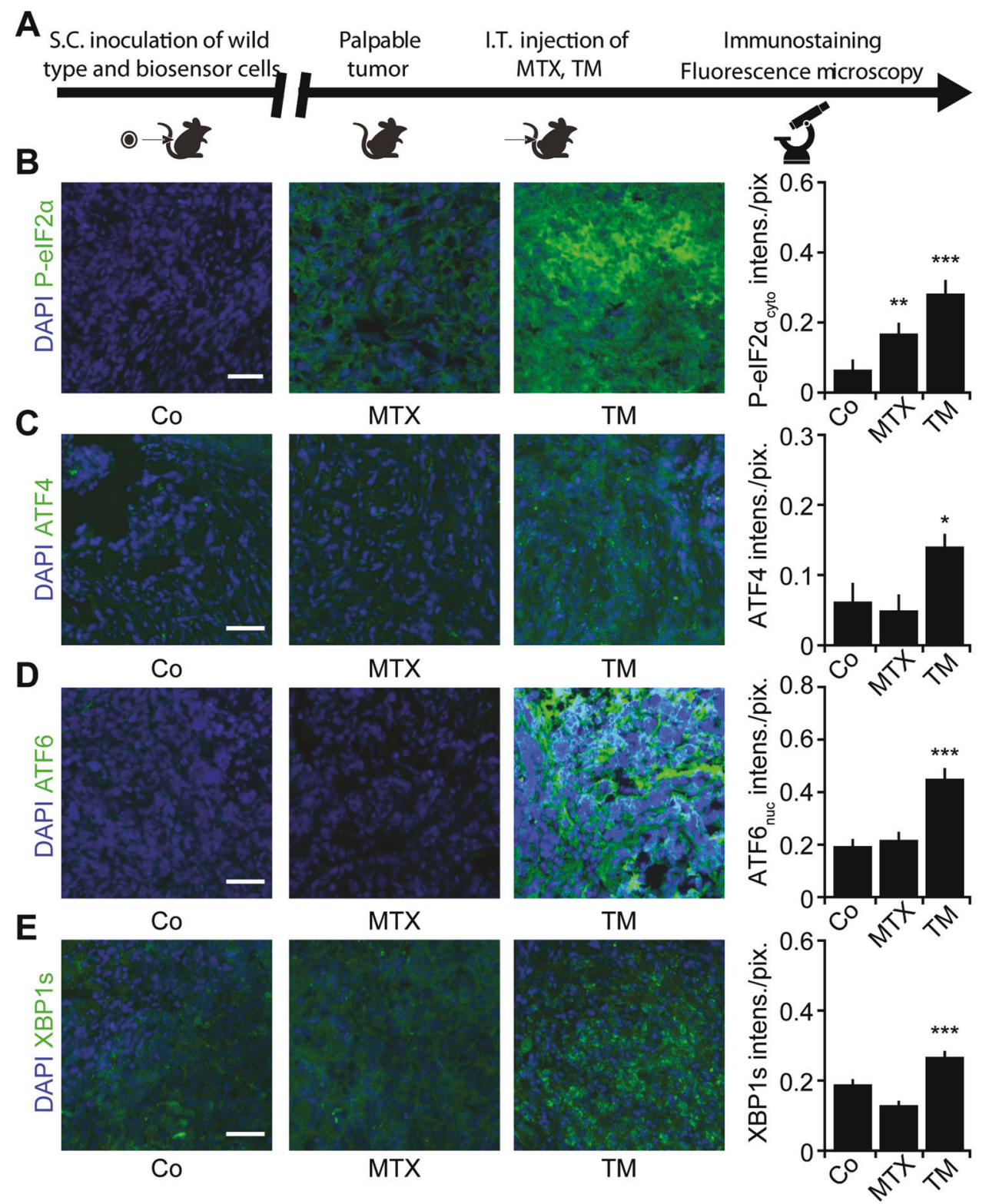

were able to stimulate the upregulation of GRP78 that typically accompanies the ER stress response, contrasting with MTX and OXA, which did not cause any increase in GRP78 protein expression (Figure S3). Of note, in contrast to CALR and PDIA3, GRP78 was not affected in its subcellular localization and hence failed to translocate to the cell surface upon treatment with ICD inducers (Figure S4 and S5). Inhibition of PERK with a specific inhibitor, GSK2606414, which acts a submicromolar concentrations, resulted in the inhibition of eIF2 $\alpha$ phosphorylation (Figure S6A) and CALR exposure (Figure S6B). Moreover, eIF2 $\alpha$ phosphorylation, which is known to be required for anthracycline-induced CALR exposure [39, 45], was required for autophagy induction (Figure S7A) but not for HMGB1 release from the nuclei (Figure S7B).
Altogether, these results support the idea that eIF $2 \alpha$ phosphorylation plays a central role in some of the ICD-related phenomena.

These finding underscore the heterogeneity of ER stress responses to chemotherapeutic agents, as well as the exclusive implication of eIF $2 \alpha$ phosphorylation, but not any of the other manifestations of ER stress for immunogenic CALR exposure.

\section{Functional interference between ICD-inducing chemotherapeutics and full activation of the ER stress response}

Intrigued by the activation of just one ER stress-linked parameter (eIF2 $\alpha$ phosphorylation) by anthracyclines, we 
Fig. 4 Correlation of cell stress and cell death

parametersCorrelation matrix of endoplasmic reticulum stress parameters including the phosphorylation of eIF $2 \alpha$ (by phosphoneoepitope-specific antibody), the translational activation of ATF4, the formation of G3BP-containing stress granules, the alternative splicing of XBP1, the nuclear translocation of ATF6, as well as cell stress and cell death parameters such as viability, the cytoplasmic translocation of HMGB1, the intracellular ATP concentration (ATP IC), calreticulin (CALR) exposure and autophagy in response to a chemical library that entails commonly used anti neoplastic agents (as detailed in the material and methods) at a final concentration of $3 \mu \mathrm{M}$. Viability was assessed by counting adherent cells with a normal nuclear morphology. Human osteosarcoma parental U2OS were treated $6 \mathrm{~h}$ and P-eIF $2 \alpha$ was obtained by immunofluorescence staining, depicted as percentage of cells with detectable cytoplasmic fluorescence. Calreticulin exposure was determined by

immunofluorescence staining and flow cytometry and is expressed as the percentage of calreticulin positive, DAPI negative (CALR $^{+}$ DAPI $^{-}$) cells. Parental U2OS were treated for $24 \mathrm{~h}$, and the average cytoplasmic HMGB1 intensity was determined by immunofluorescence staining. Parental U2OS were treated for $8 \mathrm{~h}, 16 \mathrm{~h}$ and $24 \mathrm{~h}$ to determine ATP release by quinacrine staining. The correlation was performed using a Pearson test and data are expressed as $\mathrm{R}$ coefficient. $* p<0.05, * * p<$ $0.01, * * * p<0.001$. Data are shown in a color coded diagram in which the presence or absence of positive (red) and negative (blue) correlations among the parameters is depicted along with the $p$ values of such correlations. See Figure S1 for original matrix and Table S1 for raw data
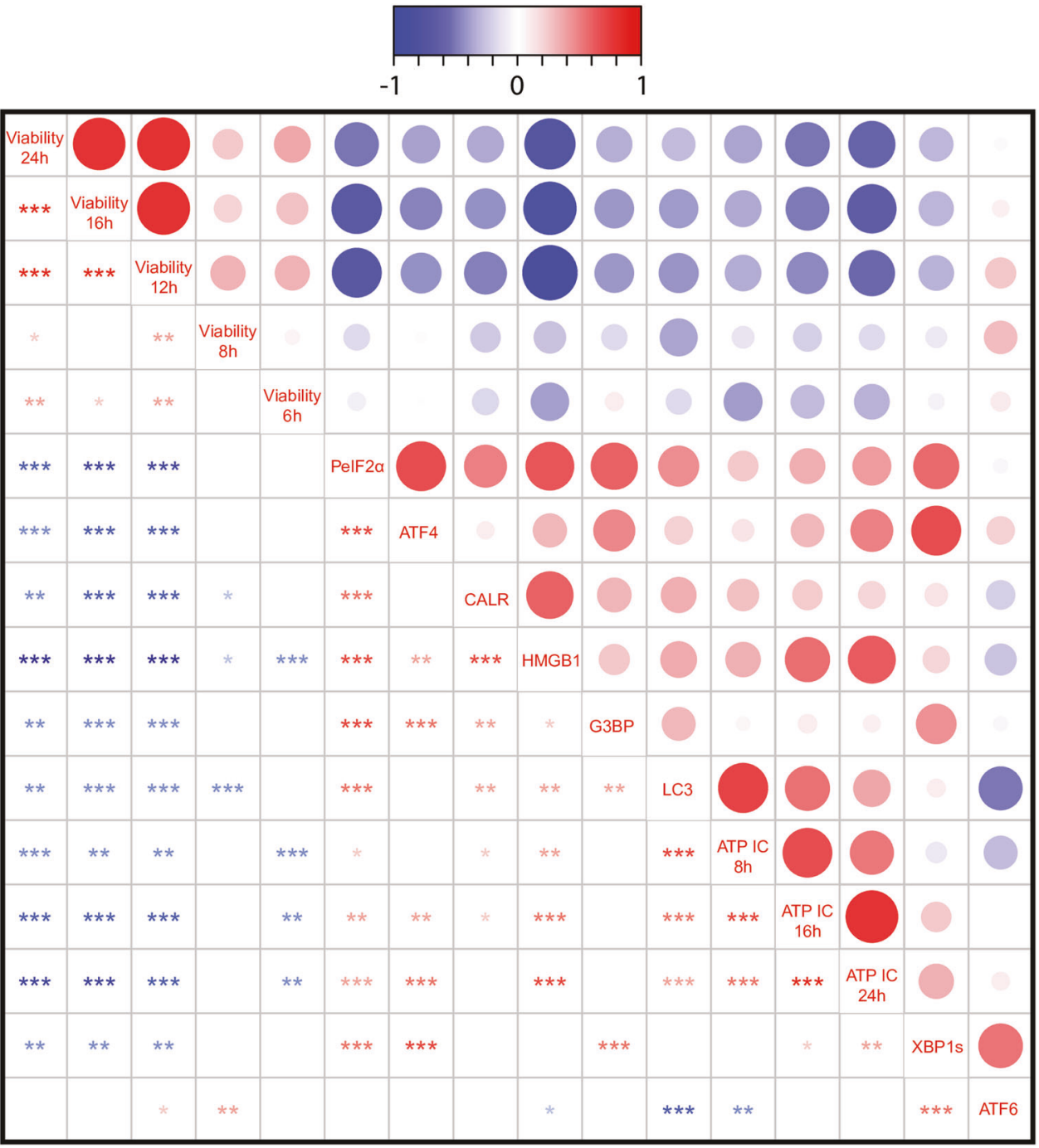

investigated the functional crosstalk between chemotherapeutic-induced stress responses and that provoked by the classical ER stressor tunicamycin. While tunicamycin was unable to induce CALR exposure, oxaliplatin and mitoxantrone readily stimulated this phenomenon. Tunicamycin, however, inhibited oxaliplatin and 


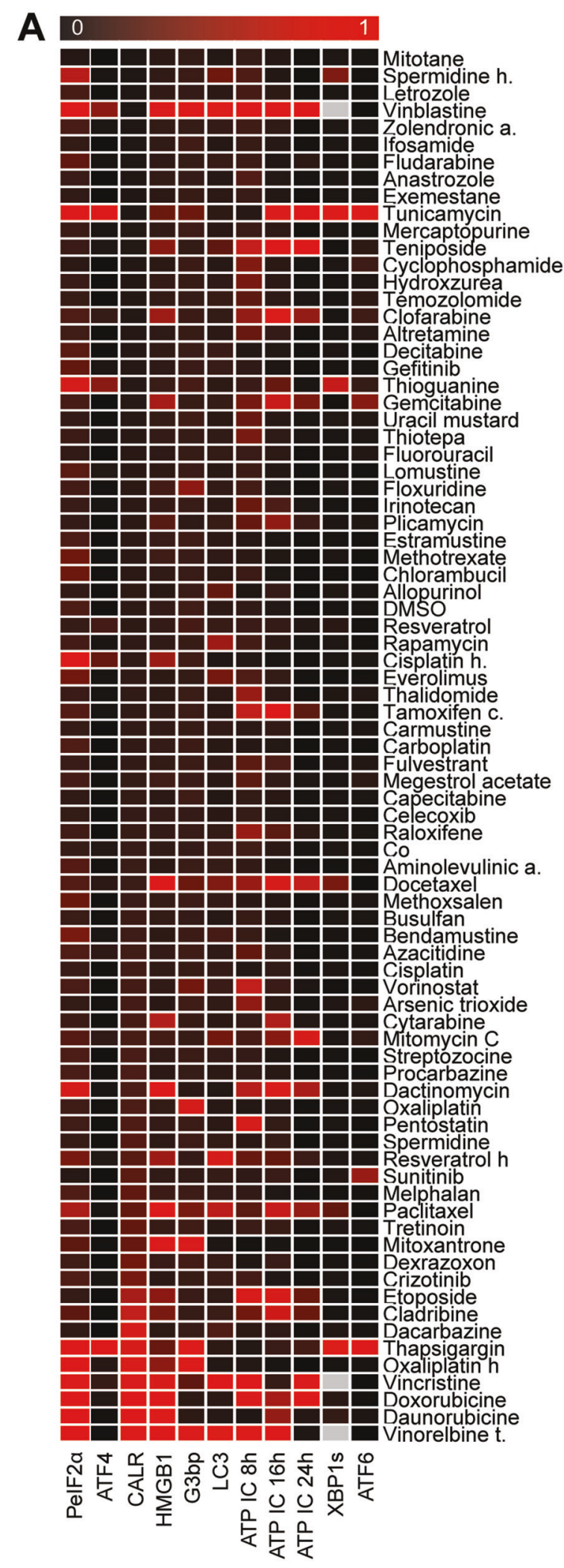

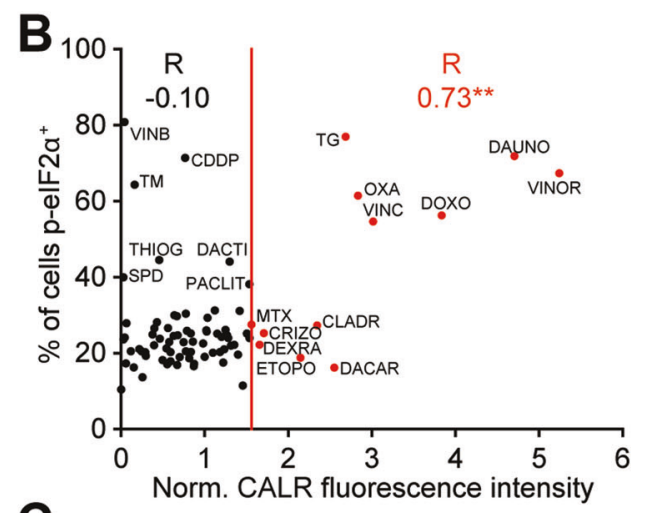
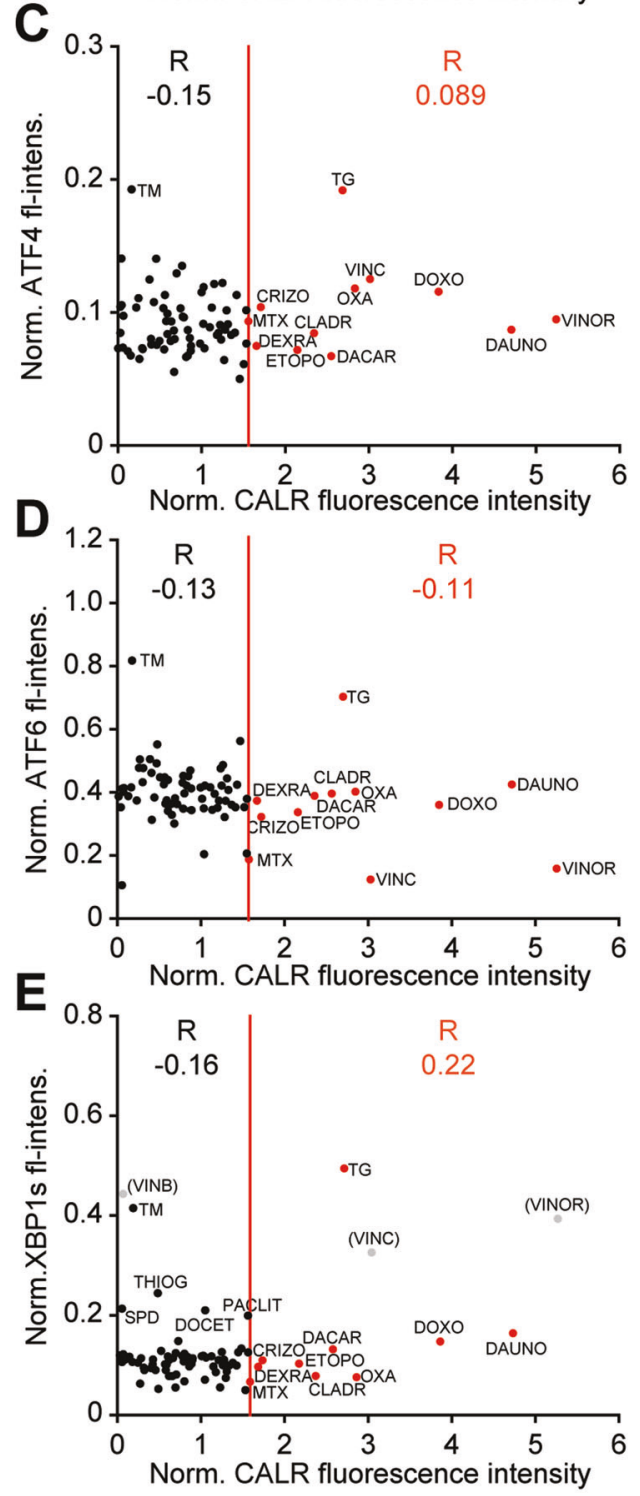
Fig. 5 CALR exposure depends on eIF $2 \alpha$ phosphorylation yet is independent of the ER stressa, $\mathbf{b}, \mathbf{c}, \mathbf{d}, \mathbf{e}$ U2OS cells were treated with a chemical library that entails commonly used anti neoplastic agents (as detailed in the material and methods) at $3 \mu \mathrm{M}$, unless otherwise specified ( $h=$ high concentration: cisplatin, $150 \mu \mathrm{M}$; oxaliplatin, 500 $\mu \mathrm{M}$; resveratrol, $50 \mu \mathrm{M}$; spermidine, $50 \mu \mathrm{M})$. U2OS cells were treated for $6 \mathrm{~h}$, and P-eIF $2 \alpha$ was detected by immunostaining. U2OS cells were treated for $24 \mathrm{~h}$, and HMGB1 was detected by immunostaining. U2OS stably expressing GFP-ATF6, pSMALB-ATF4.5rep, or XBP1 $\triangle$ DBD-venus, GFP-LC3 and G3BP-GFP have been treated for $6 \mathrm{~h}, 12 \mathrm{~h}, 12 \mathrm{~h}, 8 \mathrm{~h}$ and $12 \mathrm{~h}$, respectively (except G3BP-GFP that were treated with MTX and OXA for $24 \mathrm{~h}$ ). For the detection of surfaceexposed CALR, U2OS cells were treated for $6 \mathrm{~h}$ and CALR was detected on $\mathrm{DAPI}^{-}$cells by immunostaining and flowcytometry. Data are depicted as normalized means \pm SD of triplicates from one representative out of three experiments. Data are depicted as means \pm SD of quadruplicates from one representative out of three experiments. a Heatmap. Black and red values indicate negative and positive effects respectively (Raw data normalized with sigmoidal scaling). See Table S1 for raw data. a, e For XBP1, the results for vinca-alkaloids are not been taken into account (gray rectangles or brackets) because they were not confirmed by alternative methods (cf. Fig. 7h). The correlation was quantified using the Pearson test and data are expressed as $\mathrm{R}$ coefficients. $* p<0.05, * * p<0.01$, $* * * p<0.001$

mitoxantrone-induced CALR exposure (Fig. 6a, b). Conversely, mitoxantrone was able to prevent the activation of ATF4, CHOP, ATF6 and XBP1s by tunicamycin (Fig. 6c-g), as determined by the panoply of cell-biological or biochemical methods detailed above (see Figs. 1-2). In the next step, we performed a screen to identify anticancer agents that would be able to prevent tunicamycin-elicited ER stress responses. Mitoxantrone turned out to be the sole agent capable of preventing activation of the three arms of the ER stress response, as determined by biosensor cell lines measuring the expression of ATF4, the relocalization of ATF6, and the IRE1-mediated splicing of XBP1s (Fig. 6c-h, Table S2). Other anthracyclines (such as daunorubicin and doxorubicin) only inhibited XBP1s activation, as did the transcriptional inhibitor actinomycin D (dactinomycin). The platinum-based compounds cisplatin and oxaliplatin undistinguishable inhibited both ATF4 and XBP1s activation, yet did not affect ATF6 relocation from the ER to the Golgi and nuclei (Fig. 6h).

Altogether, these results support the notion that those agents that we have characterized as particularly strong ICD inducers, namely, anthracyclines and oxaliplatin, may not only fail to activate a full ER stress response but actually inhibit such a response.

\section{Hyperploidy-associated signs of ER stress}

Next, we investigated the relationship between CALR exposure and ER stress in the context of hyperploidy, knowing that hyperploid cancer cells tend to spontaneously expose CALR on their surface [16, 46]. Accordingly, EL4
A

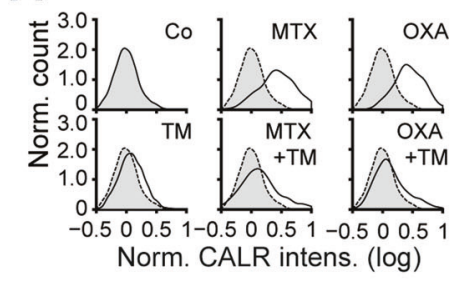

C
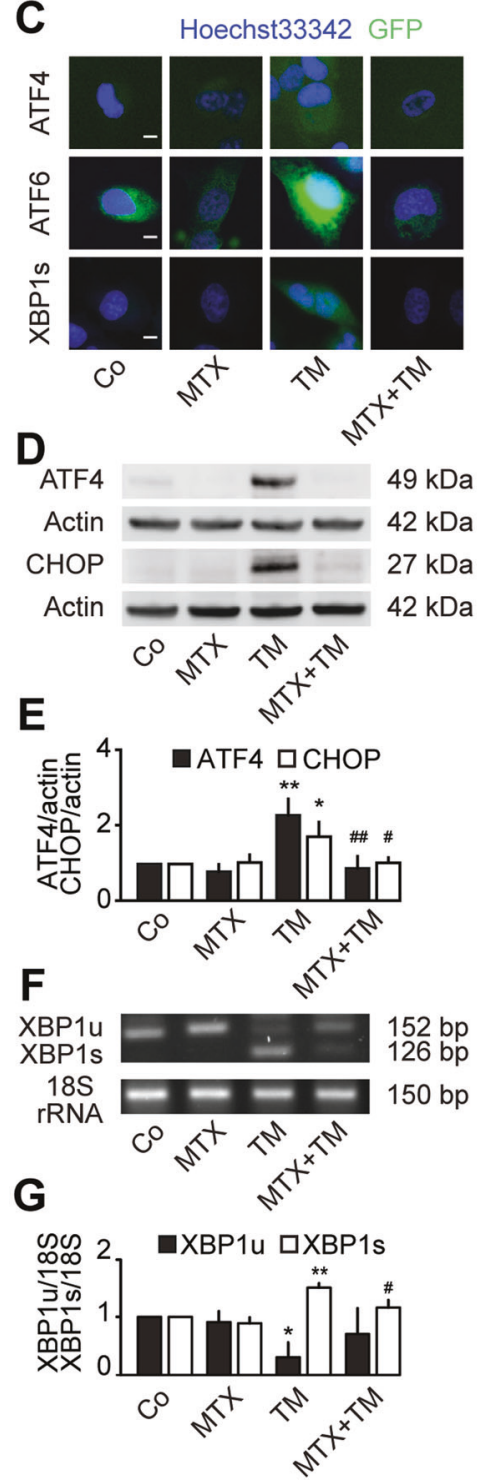

lymphoma cells that had been rendered hyperploid by transient exposure to nocodazole, followed by cytofluorometric purification of cells with a $>4 n$ DNA content and isolation of clones, exhibited higher levels of calreticulin than parental EL4 cells. If such cells were passaged through immunodeficient mice $N S G$ mice (which lack adaptive immunity due to the absence of $\mathrm{B}$ and $\mathrm{T}$ cells), they maintained their hyperploid cells and high CALR exposure. However, when passaged through immunocompetent mice
B

H

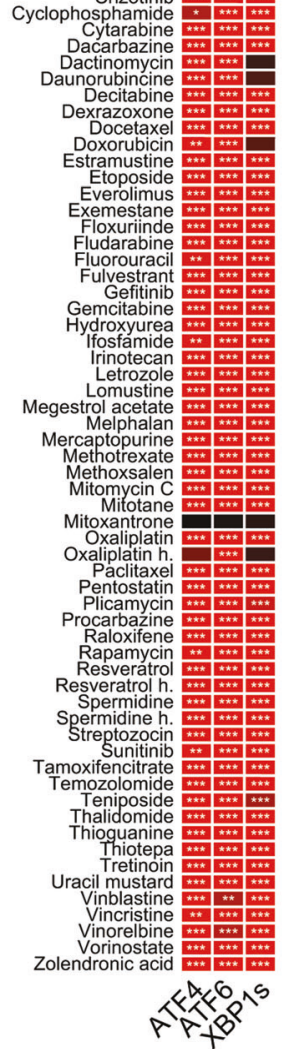


Fig. 6 Mutually exclusive induction of ER stress and calreticulin exposurea, b U2OS cells were treated with mitoxantrone (MTX, $3 \mu \mathrm{M}$ ) or oxaliplatin (OXA, $500 \mu \mathrm{M})$ in the presence or absence of tunicamycin (TM, $3 \mu \mathrm{M})$. CALR exposure was detected by immunostaining and cytometry. Representative histograms of surface CALR and means $\pm S D$ of triplicates from one representative out of three experiments are depicted. Data were compared with Student's $t$-test. $* p<0.05, * * p<0.01, * * * p<0.001$. c U2OS stably expressing GFPATF6, pSMALB-ATF4.5rep, or XBP1 $\triangle \mathrm{DBD}$-venus were treated for $6 \mathrm{~h}, 12 \mathrm{~h}$, and $12 \mathrm{~h}$, respectively with mitoxantrone (MTX, $3 \mu \mathrm{M}$ ) or tunicamycin (TM, $3 \mu \mathrm{M}$ ) or both. Representative images (scale bar equals $10 \mu \mathrm{m}$ ) are shown. d, e U2OS cells were treated with MTX (2 $\mu \mathrm{M})$ in the presence or the absence of TM $(3 \mu \mathrm{M})$ and the expression of ATF4 and CHOP was assessed by specific antibodies. Representative immunoblots (d) and densitometry data (e) shown as mean value \pm SD of three independent experiments are depicted $* p<0.05$, $* * p<0.01$, $* * * p<0.001(\mathbf{f}, \mathbf{g}) \mathrm{U} 2 \mathrm{OS}$ cells were treated with MTX $(1 \mu \mathrm{M})$ in the presence or the absence of TM $(3 \mu \mathrm{M})$, and total RNA was isolated. Reverse transcriptase polymerase chain reaction (RT-PCR) was performed with primers specific for human XBP1s. PCR products were separated by gel electrophoresis. Representative images (f) and densitometry data $(\mathbf{g})$ shown as mean value \pm SD of three independent experiments are depicted. Samples were compared using Student's $t$ test $(* p<0.05, * * p<0.01, * * * p<0.001)$. h U2OS cells were treated with a chemical library that contains commonly used anti neoplastic agents (as detailed in the Material and Methods) at $3 \mu \mathrm{M}$ unless otherwise specified ( $h=$ high concentration: cisplatin, $150 \mu \mathrm{M}$; oxaliplatin, $500 \mu \mathrm{M}$; resveratrol, $50 \mu \mathrm{M}$; spermidine, $50 \mu \mathrm{M}$ ) in the presence of TM $(3 \mu \mathrm{M})$. U2OS stably expressing GFP-ATF6, pSMALBATF4.5rep, or XBP1 $\mathrm{DBD}$-venus were treated for $6 \mathrm{~h}, 12 \mathrm{~h}$, and $12 \mathrm{~h}$, respectively and GFP and venus were detected by fluorescence microscopy. Heatmap. Red and black values indicate the absence or presence of inhibition, respectively (Raw data normalized with sigmoidal scaling). Results are expressed as mean \pm SD. Data were compared with $t$-test. $* p<0.05, * * p<0.01, * * * p<0.001$. See Table S2 for raw data

they reduced their DNA content and the level of CALR exposure (Fig. 7a). During acute tetraploidization of U2OS cells eIF2 $\alpha$ was hyperphosphorylated yet no additional signs of ER stress were detected (Fig. 7d, f and h). Similarly parental HCT116 cells readily mounted the full panoply of ER stress responses to thapsigargin. In contrast, hyperploid clones (T1 and T2) as well as parental HCT116 cells only exhibited eIF2 $\alpha$ hyperphosphorylation but not any other signs of ER stress including activation of ATF4 and XBP1 (Fig. 7e, g and i). Transcriptome profiling followed by bioinformatic analysis confirmed that XBP1 and ATF6 target genes were not significantly up or downregulated in hyperploid EL4 cells or after the passage of such cells through mice with different levels of immune competence (Fig. 7b). However, ATF4 target genes were mostly downregulated in hyperploid cells and were expressed at a higher level in tumors that had undergone immunoselection in immunocompetent mice than in tumors that were recovered from immunodeficient mice (Fig. $7 \mathrm{~b}, \mathrm{c}$ ).

These results provide yet another example in which CALR exposure appears to be exclusively linked to eIF $2 \alpha$ phosphorylation, but not to any other facet of the ER stress response.

\section{Prediction of ICD induction based on physicochemical and structural drug characteristics}

In the course of this research project, we generated a large database describing the cellular response to a panel of clinically used anticancer drugs (Fig. 4). Previous studies revealed that many of the cellular effects of several ICD inducers are not linked to their 'on-target' effects (such as DNA damage for anthracyclines and oxaliplatin) but rather must be attributed to 'off-target' effects that may be linked to their broad physicochemical characteristics $[10,39]$. We attempted to delineate a machine learning approach to identify the known physicochemical/molecular characteristics of pharmaceutical compounds that best correlate with the biological characteristics that define ICD, while attempting to reduce the complexity of both data sets (Fig. 8a). The 343 physicochemical values that can be retrieved for each of the compounds in the library ( 24 from Pubchem database, https://pubchem.ncbi.nlm.nih.gov, and 319 calculated using the Chemistry Development Kit CDK, https://github.com/cdk) were reduced to 93 relevant values, each of which was positively or negatively weighted in an equation (Fig. 8c, S9, Table S3), leading to the calculation of an 'ICD score'. This simplified score was constrained to optimally fit the sum of five biological signs of ICD, namely, eIF2 $\alpha$ phosphorylation, CALR exposure, HMGB1 exodus, formation of G3BP stress granules and LC3 puncta (Fig. 8b). The inclusion of additional information on biological responses did not further improve the correlation $(R=0.78)$ between biological and physicochemical characteristics explaining ICD. In the next step, we applied the ICD score to two chemical libraries in which ICD inducers have been identified in the past by biological screening methods, i.e., the collection of Food and Drug Administration (FDA)-approved drugs [28] and the NCI Mechanistic Diversity Set [29]. Importantly, this approach allowed to class most bona fide ICD inducers in the upper $15 \%$ quantile, leading to a significant $(p<0.01$, Kolmogorov-Smirnov test) enrichment of ICD inducers (Fig. 8d, e, Figure S8).

Altogether these results validate the possibility to use structural and physicochemical information on individual drugs to predict whether they induce ICD with a significant probability. Moreover, it is noteworthy that eIF $2 \alpha$ phosphorylation itself, but none of the other hallmarks of ER stress, and several features downstream of eIF $2 \alpha$ phosphorylation including CALR exposure, stress granule formation and autophagy appear particularly well associated with ICD induction. 


\section{Discussion}

The results included in this article lend strong support to the idea that the phosphorylation of eIF $2 \alpha$ is a pathognomonic hallmark of ICD. Although we had observed in the past that only eIF $2 \alpha$ phosphorylation but neither of the other two arms of the ER stress response (namely the IRE1/XBP1 axis and the ATF6 pathway) are required for the ICD-related induction of CALR exposure by anthracyclines and oxaliplatin [39], it appears surprising that these latter two arms are actually not induced in the context of ICD, neither in vitro (in cultured human cells exposed to a collection of
A
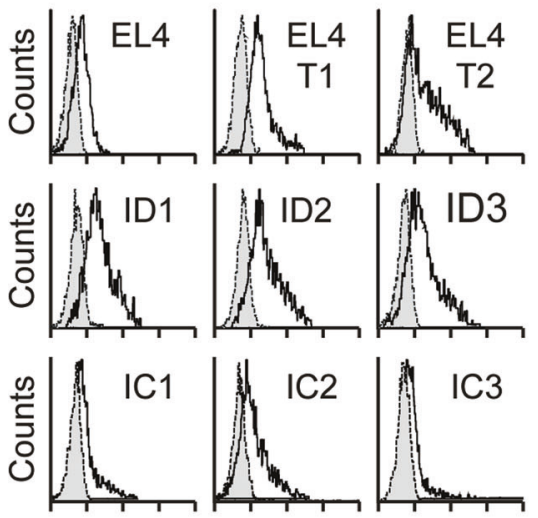

012340123401234

D

CALR-AF488 (log mean fl.)

P-elF2a $-\cdots-\cdots-\cdots \mathrm{kDa}$ Actin $-42 \mathrm{kDa}$

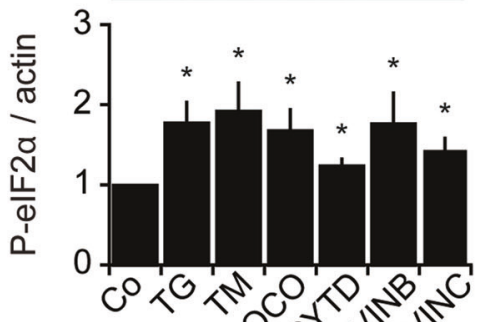

F
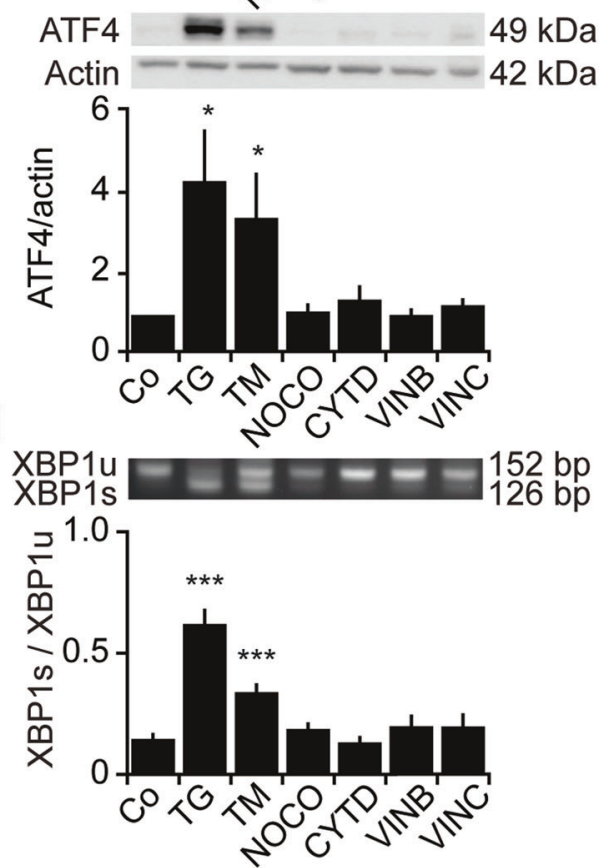

B

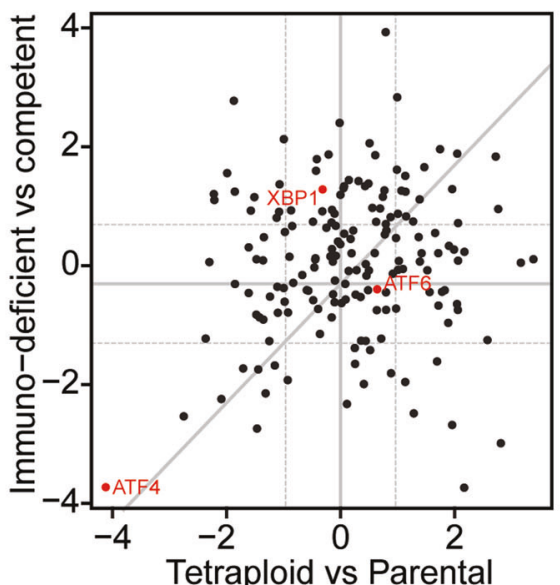

E
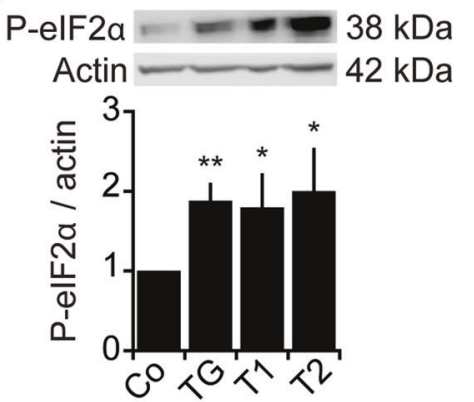

G

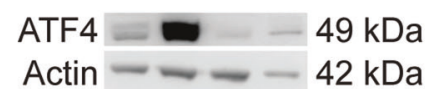

|
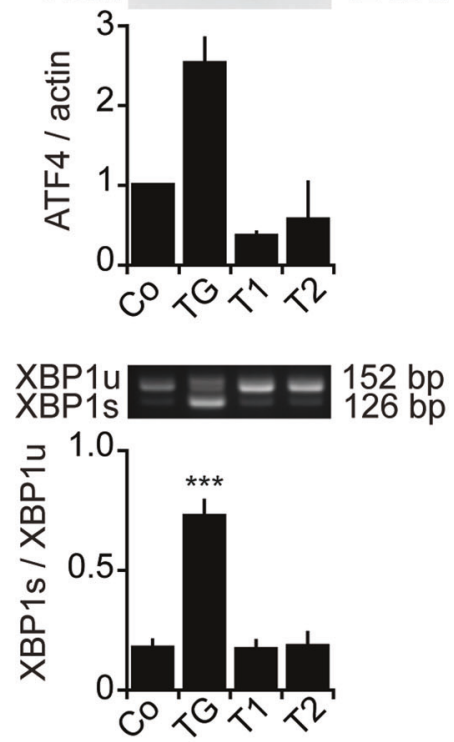

C

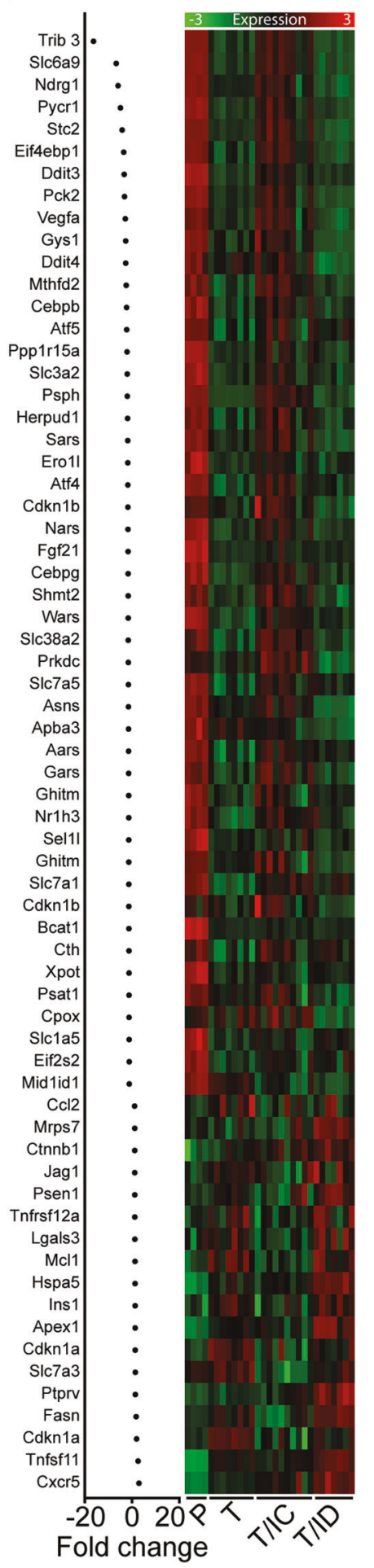


Fig. 7 Hyperploidy-associated signs of ER stressa CALR exposure induced by hyperploidy. Parental EL4 cells and its two tetraploid derivatives (EL4-T1 and EL4-T2) were cultured in vitro (upper line) or passaged through immunodeficient (ID) mice or immunocompetent mice (IC). These cells were the stained to assess calreticulin exposure by indirect immunofluorescence (gray curves indicate isotype controls). b Scatter plot representation of transcription factors (including ATF4, ATF6, XBP1) that, according to bioinformatic analyses, correlate with differences in the transcriptome between parental and tetraploid cells cultured in vitro (abscisse) or that between tetraploid clones passaged through ID vs. IC mice (ordinate). Transcription factor activities are expressed as the $z$-scores obtained by "IPA Upstream Regulator Analysis". c Expression of selected ATF4 target genes. For each gene, the log fold change between hyperploid cells compared to parental cells is shown, Moreover, expression levels are shown in a heat map for each sample. ER stress activation was measured during acute tetraploidization $(\mathbf{d}, \mathbf{f}, \mathbf{h})$ of U2OS cells or longterm tetraploidization of HCT116 cells $(\mathbf{e}, \mathbf{g}, \mathbf{i})$. U2OS cells were left untreated (control, Co) or treated with thapsigargin (TG, $3 \mu \mathrm{M}$ ), tunicamycin (TM, $3 \mu \mathrm{M})$, nocodazole (Noco, $100 \mathrm{nM}$ ), cytochalasin D (CytD) $1.2 \mu \mathrm{M}$, vinblastine (Vinb) $3 \mu \mathrm{M}$ or vincristine (Vinc) $3 \mu \mathrm{M}$ for $6 \mathrm{~h}(\mathrm{P}-\mathrm{eIF} 2 \alpha)$ or $12 \mathrm{~h}$ (ATF4). Parental HCT116 cells were left untreated $(\mathrm{Co})$ or treated with thapsigargin (TG, $3 \mu \mathrm{M}, 6 \mathrm{~h})$ and compared to two tetraploid clones (T1, T2). The indicated signs of ER stress were determined by immunoblot $(\mathbf{d}-\mathbf{g})$ or RT-PCR $(\mathbf{h}, \mathbf{i})$. Representative blots are shown. Densitometric quantifications are means \pm SEM of at least three experiments and are analyzed by means of the Student's $t$-test $\left({ }^{*} p<0.05,{ }^{*} p<0.01, * * * p<0.001\right)$

distinct chemotherapeutic agents) nor in vivo (in chemotherapy-treated cancers and in polyploid, immunogenic tumors). Indeed, using less sophisticated technologies for the assessment of ATF6 and XBP1 activation, we previously failed to document the "split" ER stress response induced by ICD inducers [28]. Moreover, it is rather unexpected that ATF4 is not activated in the context of ICD, downstream of eIF $2 \alpha$ phosphorylation, as this should occur in canonical ER stress response [47]. The molecular mechanisms that explain how eIF2 $\alpha$ phosphorylation can be uncoupled from other hallmarks of ICD are thus far elusive. Usually, the three arms of the ER stress response are activated in a highly coordinated fashion because the occupancy of the chaperone GRP78 by unfolded proteins causes the close-to-simultaneous release of PERK/EIF2AK3, IRE1 and ATF6 from GRP78 [48]. We have previously described that PERK was required for eIF $2 \alpha$ phosphorylation in the context of ICD induced by oxaliplatin [39] and that anthracyclines were able to dissociate the interaction between PP1 and GADD34, thereby reducing eIF2 $\alpha$ dephosphorylation [49]. Hence, eIF2 $\alpha$ phosphorylation induced by chemotherapeutic agents may be catalyzed through an imbalance of the action of kinases and phosphatases, independently from ER stress. This possibility requires further in-depth investigation. Indeed, at this point it is not known whether an increase in the enzymatic activity of the eIF2 $\alpha$ kinase or a decrease in the activity of the phosphatase (or both) account for the hyperphosphorylation of eIF2 $\alpha$. Irrespective of this unresolved question, it appears that translation is arrested upon addition of anthracyclines or oxaliplatin to cells, thought the signs of full-blown endoplasmic reticulum response including its final outcome (which includes the upregulation of GRP78) do no manifest.

Importantly, some particularly efficient ICD inducers such as anthracyclines actively inhibit facets of the ER stress response induced by tunicamycin. Although the mechanisms of this suppressive effect remain elusive, they are apparently not important for ICD induction at large, because other ICD inducers, such as oxaliplatin and vincaalkaloids, failed to interfere with ER stress responses, while cisplatin (which does not induce ICD) did so. Moreover, the algorithm designed to identify the biological characteristics of ICD inducers could dispense with the information on ER stress-modulatory drug effects, with the sole and important exception of eIF2 $\alpha$ phosphorylation. Irrespective of the mechanistic uncertainties, it appears that eIF2 $\alpha$ phosphorylation provides a central biomarker for diagnosing immunogenicity in the context of ICD. Accordingly, eIF $2 \alpha$ phosphorylation was observed in vivo, shortly after chemotherapy with anthracyclines (Fig. 3) as well as in polyploid tumor cells, both in mice (Fig. 7) and in tumor samples from breast cancer patients [16]. eIF $2 \alpha$ phosphorylation apparently can be measured as a surrogate marker of CALR exposure in non-small cell lung cancer and acute myeloid leukemia [30, 31]. In all these pathologies, elevated eIF $2 \alpha$ phosphorylation is a positive prognostic feature [30, $31,50]$. This effect may be linked to the fact that eIF $2 \alpha$ phosphorylation contributes to both CALR exposure and autophagy, which both concur to render cell death immunogenic.

The compilation of multiple cellular stress responses induced by a battery of anticancer drugs allowed us to compute an algorithm that predicts ICD characteristics based on biological effects including eIF $2 \alpha$ phosphorylation and that correlates with the physicochemical characteristics of such drugs. Importantly, the results that were obtained in the discovery data set (with a collection of FDA-approved anticancer cytotoxicants, as well as a few positive controls such as thapsigargin and tunicamycin for ER stress induction and spermidine, rapamycin and resveratrol for autophagy induction) could be validated in two additional data sets, namely the collection of all FDA-approved drugs (beyond the antineoplastic agents) and a library of chemical compounds that are being characterized at the NCI for possible anticancer effects. Based on this algorithm, it appears possible to pre-select compounds with putative proICD properties from chemical libraries, thereby reducing the cost of screening campaigns designed to identify ICD inducers. However, the predictive value of this algorithm is not absolute, because, for example, spicamycin/septacidin, 
A

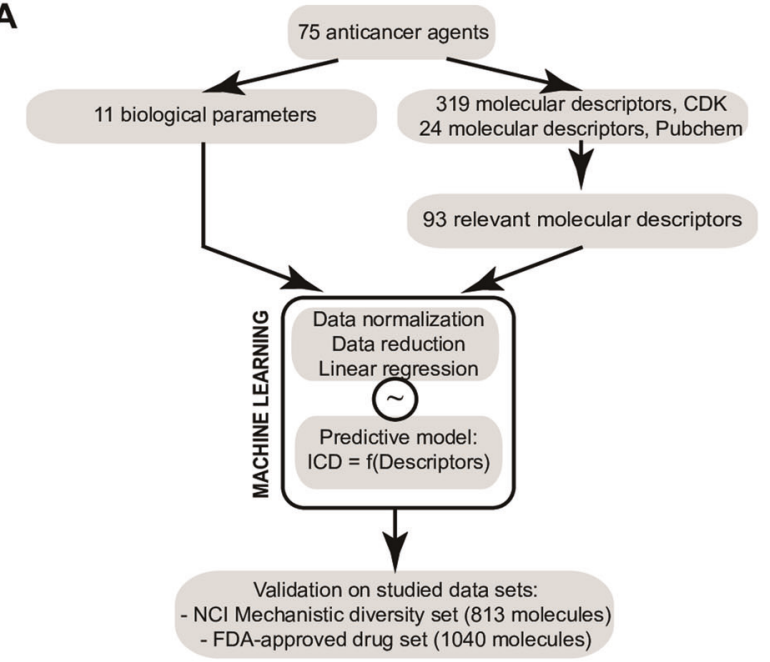

C

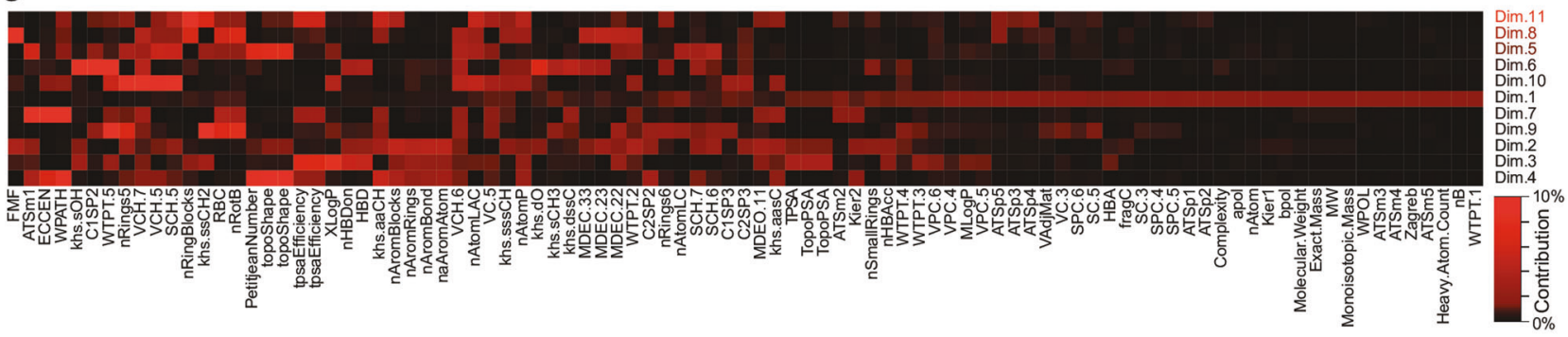

B

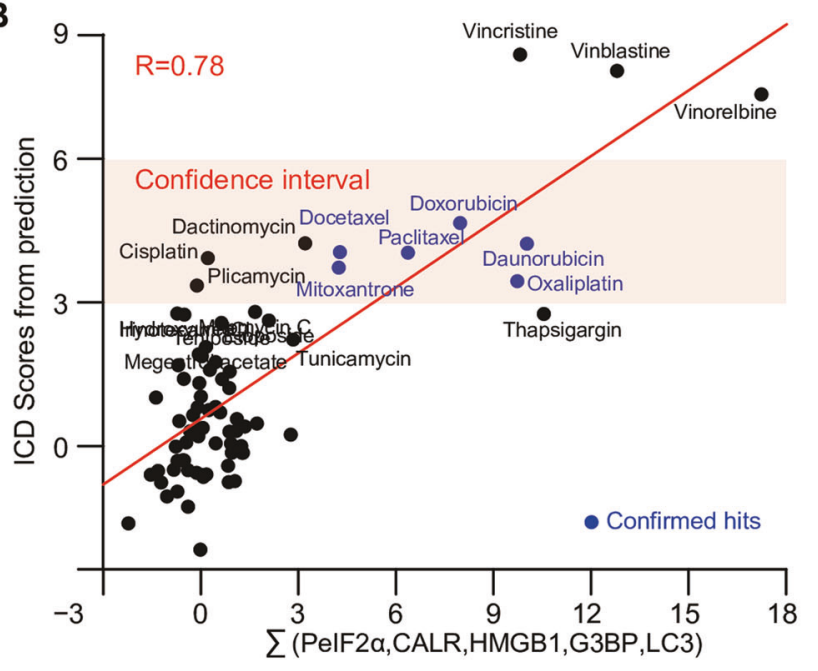

D

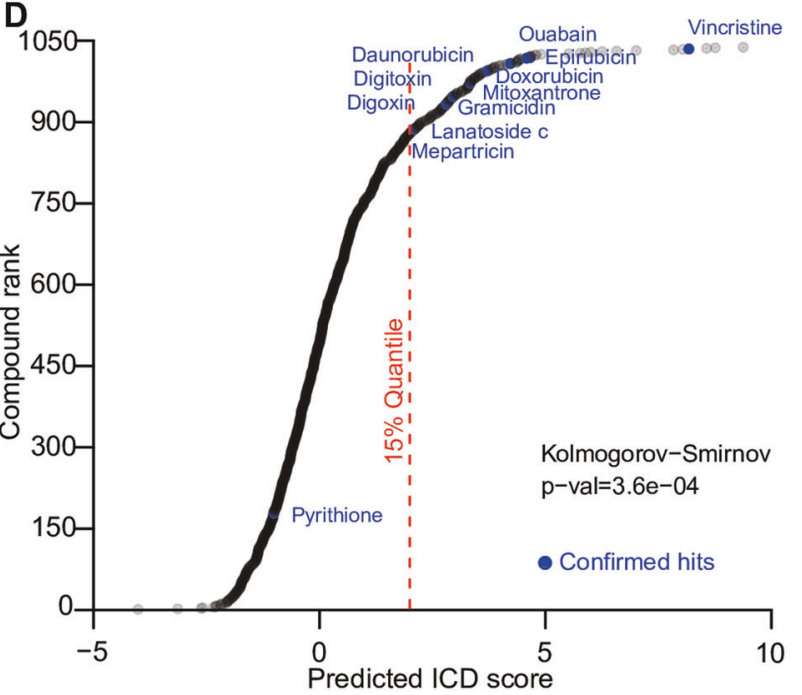

Fig. 8 Chemical descriptors and immunogenic cell death predictiona The main steps leading to the construction of a mathematical model for the prediction of immunogenic cell death (ICD) are depicted as flowchart. The prediction model was built by machine learning, performing an optimized Principal Component Regression (PCR) between the 11 biological parameters and the 93 chemical descriptors available for each agent from the training set. The relevant chemical descriptors were retained from a total of 343 descriptors, either retrieved from PubChem (https://pubchem.ncbi.nlm.nih.gov) or calculated using the Chemistry Development Kit (https://github.com/cdk). The generated model was validated on two published experimental data sets by comparing the distribution of predicted scores among experimental hits (excluding those used for model construction) with remaining compounds. b An optimized model was obtained by summing 5 out of 11 biological parameters as ICD indicators, including eIF $2 \alpha$ phosphorylation. The predicted scores obtained from the model are plotted against the scores

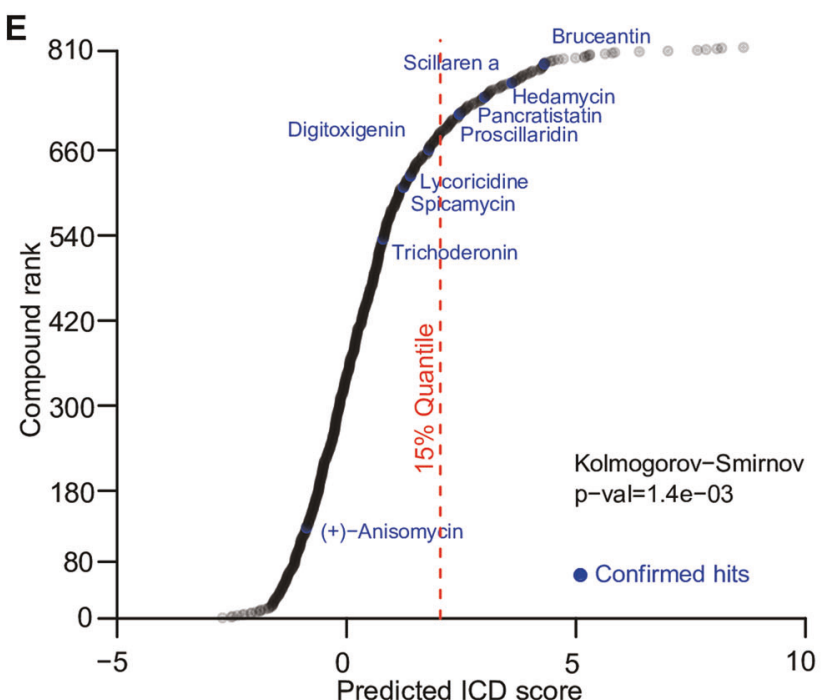

obtained experimentally. The linear regression between predicted and experimental scores is indicated (red line) and the Pearson correlation coefficient $\mathrm{R}$ is reported. Compounds indicated in blue are hits validated in vitro and were used as a constraint for model optimization. Other indicated compounds had a predicted score higher than 2. Upon outlier exclusion, we defined an arbitrary confidence interval ranging from ICD score 3 to 6 . $\mathbf{c}$ The contribution of each descriptor composing the 11 dimensions from principal component analysis (PCA) projection used in model are reported as heatmap. Dimensions are ordered according to their decreasing weight in linear regression. d, e Predicted scores for compounds present in the FDA-approved drug library (1040 agents, d) and NCI Mechanistic diversity set (813 agents, e) were calculated and are reported on the graphs. The results of the Kolmogorov-Smirnov statistical test between the scores of experimental ICD inducers (in blue) and remaining compounds are reported on the graphs. The red dashed line represents the top $15 \%$ quantile of the scores 
which is a validated ICD inducer [29], was not found in the upper $15 \%$ quantile of the screening, while many compounds that are apparently devoid of ICD activity are classified as positive. Hence, if the question arises whether individual compounds induce ICD or not, their physicochemical characteristics do not allow to make accurate predictions, and experimentation is required to resolve the problem. Nonetheless, we believe that the algorithm that predicts ICD inducer with a certain probability will be useful in future screening efforts to identify ICD inducers in large chemical libraries. Indeed, this algorithm, which is solely based on known molecular characteristics, can be used to "filter" such libraries to select only those compounds that have a high chance to activate anticancer immune responses, thus reducing the cost of the screening effort.

Altogether, the present results support the idea that $\operatorname{IF} 2 \alpha$ phosphorylation constitutes a convenient biomarker that predicts immunogenicity of cancer cells in the context of iatrogenic stress.

\section{Materials and methods}

\section{Cell culture}

Human osteosarcoma U2OS cells and mouse embryonic fibroblasts (MEF) cells were cultured in Dulbecco's modified Eagle's medium, human colon cancer HCT 116 cells were cultured in McCoy's medium and mouse lymphoma EL4 cells were cultured in RPMI 1640. All media (Thermo Fisher Scientific, Carlsbad, CA, USA) were supplemented with $10 \%$ fetal bovine serum $\left(\right.$ Gibco $^{\circledR}$ by Life Technologies, Carlsbad, CA, USA), $1 \%$ nonessential amino acids (Thermo Fisher Scientific), 1\% HEPES (Thermo Fisher Scientific) and 1\% penicillin/streptomycin (Thermo Fisher Scientific) in a humidified incubator with $5 \% \mathrm{CO}_{2}$ at $37^{\circ} \mathrm{C}$

\section{Antibodies}

Rabbit monoclonal antibodies against HMGB1 (\#ab79823), phospho-eIF2 $\alpha$ (Ser51) (\#ab32157), CALR (\#ab2907), and mouse monoclonal antibody against ATF6 (\#ab122897), PDIA3/ERp57 (\#ab13506) and rabbit polyclonal against GRP78/BiP (\#ab21685) were purchased from Abcam (Cambridge, UK). Rabbit polyclonal antibody against eIF2 $\alpha$ (\#9722 S), LC3B (\#2775), rabbit monoclonal antibody ATF4 (\#11815) and mouse monoclonal antibody against CHOP (clone L63F7; \#2895) were from Cell Signaling Technology (Danvers, MA, USA). Mouse monoclonal antibody against XBP1s (\#658802) was obtained from BioLegend (San Diego, CA, USA).

\section{Plasmid construction and transfection}

U2OS cells stably expressing GFP-LC3, U2OS G3BP-GFP or XBP1 $\triangle \mathrm{DBD}$-venus were generated by our group in the past [51, 16]. U2OS cells stably expressing GFP-ATF6 were obtained from Dr. Silke Nock and Prof. Dr. Peter Walter (California University), and purchased from Thermo Scientific (084_01). U2OS cells stably expressing pSMALB-ATF4.5rep (ATF4 reporter, GFP expressed upon eIF2 $\alpha$ phosphorylation) were obtained by lentiviral transduction followed by FACS-assisted cloning. Lentivirus was produced in HEK 293FT cells (Thermo Fisher Scientific) transfected with an pSMALB-ATF4.5rep encoding construct together with the ViraPower lentiviral packaging mix from Thermo Fisher Scientific by means of Lipofectamine $2000^{\circledR}$ (Thermo Fisher Scientific) in Opti-MEM ${ }^{\text {TM }}$ (Thermo Fisher Scientific). Two days later lentiviral particles were harvested and used for transduction.

\section{Treatments}

Cells were seeded in 96-well or 384-well plates and treated with the following drugs: allopurinol (A8003); altretamine (549835); aminolevulinic acid (A7793); anastrozole (Y0001522); arsenic trioxide (311383); azacitidine (A2385); bendamustine (B5437); busulfan (B2635); capecitabinem (SML0653); carboplatin (C2538); carmustine (C0400); celecoxib (Y0001445); chlorambucil (C0253); cisplatin (C2210000); cladribine (Y0000639); clofarabine (C7495); crizotinib (PZ0191); cycloheximide (C7698); cyclophosphamide (C3250000); cytarabine (C6645); cytochalasin D (C8273); dacarbazine (Y0000733); dactinomycin (A1410); daunorubicin (D0125000); decitabine (A3656); dexrazoxone (D1446); docetaxel (01885); doxorubicin (D1515); estramustine disodium phosphate (E0407); etoposide (E2600000); everolimus (07741); exemestane (PZ0006); floxuridine (F0503); fludarabine (Y0001536); fluorouracil (F6627); fulvestrant (Y0001399); gefitinib (M72803); gemcitabine $\mathrm{HCl}$ (G6423); hydroxyurea (H8627); ifosfamide (I0060000); irinotecan (I1406); letrozole (Y0000685); lomustine (L5918); megestrol acetate (M0513); melphalan (Y0001457); mercaptopurine (852678); methotrexate (M1000000); methoxsalen (M3501); mitomycin C (M4287); mitotane (25925); mitoxantrone (M6545); nocodazole (M1404); pentostatin (SML0508); paclitaxel (T7191); plicamycin (M6891); procarbazine (SML0036); raloxifene (Y0001134); spermidine (S2626); streptozocin (S0130); sunitinib (PZ0012); tamoxifen citrate (T0014000); temozolomide (76899); teniposide (SML0609); thalidomide (T144); thapsigargin (T9033); thioguanine (A4882); thiotepa (T6069); tretinoin (PHR1187); tunicamycin (T7765); uracil mustard (S375063); vinblastine sulfate (V1377); vincristine sulfate (V0400000); vinorelbine tartrate (Y0000463); vorinostat (SML0061); zolendronic 
acid (SML0223) that were bought from Sigma-Aldrich (St. Louis, MI, USA). Oxaliplatin came from Sigma-Aldrich (Y0000271) or from Accord Healthcare (Ahmedabad, India); resveratrol (BML-FR104-0100) from ENZO Life Sciences (Farmingdale, New York, USA) or from Sigma Aldrich (R8781); rapamycin (1292) and GSK 2606414 from Tocris bioscience (Bristol, UK).

\section{Western blot analysis}

Protein extraction was conducted in RIPA buffer (\#89900; Thermo Fisher Scientific) in the presence of phosphatase and protease inhibitors (\#88669; Thermo Fisher Scientific) followed by sonication. Then protein content was measured by Bio-rad laboratory $\mathrm{DC}^{\mathrm{TM}}$ Protein Assay reagent A, B and S (\#500-0113, \#500-0114 and \#5000115). A minimum of $15 \mu \mathrm{g}$ of protein was solved in Laemmli buffer (Thermo Fisher Scientific), denaturated at $100{ }^{\circ} \mathrm{C}$ and separated by polyacrylamide gel electrophoresis using 4-12\% Bis-Tris pre-casted gels (Thermo Fisher Scientific) in MES buffer (Thermo Fisher Scientific). Following proteins were transferred to nitrocellulose membrane (Merck Millipore IPVH00010) in transfer buffer (25 mM Tris; $190 \mathrm{mM}$ glycine; $20 \%$ methanol in $\mathrm{H} 2 \mathrm{O})$ at $200 \mathrm{~mA}$ for $1.5 \mathrm{~h}$. Membranes were washed in Tris-buffered saline with Tween 20 buffer (TBST; $20 \mathrm{mM}$ Tris, pH $7.5150 \mathrm{mM} \mathrm{NaCl} 0.1 \%$ Tween 20 in $\mathrm{H}_{2} \mathrm{O}$ ) and then treated with blocking buffer $(5 \%$ bovine serum albumin (BSA) in TBST) for $1 \mathrm{~h}$. Membranes were exposed to primary antibody diluted in 5\% BSA in TBST overnight at $4{ }^{\circ} \mathrm{C}$. Following membranes were washed repeatedly with TBST and then were incubated with appropriate horseradish peroxidase-coupled secondary antibody (Southern Biotech, Birmingham, AL, USA) for $1 \mathrm{~h}$ at room temperature. Proteins were revealed with ECL (GE Healthcare, Chicago, Il, USA). Beta-actin has been measured to verify equal protein quantity in a routine fashion.

\section{Reverse transcription reaction and polymerase chain reaction}

Total RNA was extracted using the GeneJet RNA purification kit from Thermo Scientific (\#K0731). Following reverse transcription was performed with the Maxima First Strand cDNA Synthesis Kit from Thermo Scientific (\#K1671) on a minimum of $1.5 \mu \mathrm{g}$ total RNA template using the following cycle $10 \mathrm{~min}$ at $25^{\circ} \mathrm{C}$ followed by 45 min at $50{ }^{\circ} \mathrm{C}$ then $5 \mathrm{~min}$ at $85^{\circ} \mathrm{C}$. PCR was performed with a mix of $0.16 \mu \mathrm{g}$ of each primer (reverse primer for human XBP1s 5'-CCATGGGGAGATGTTCTGGAG-3'; forward primer for human XBP1s 5'-CCTGGTTGCTGAAG AGGAGG-3' from Sigma-Aldrich), a minimum of $1.5 \mu \mathrm{g}$ of cDNA and nucleotide-containing PCR Master Mix (\#4367659; Thermo Fisher Scientific) using the following program: $94^{\circ} \mathrm{C}$ for $4 \mathrm{~min}$, then 30 cycles $94^{\circ} \mathrm{C}$ for $30 \mathrm{~s}, 58$ ${ }^{\circ} \mathrm{C}$ for $45 \mathrm{~s}, 72{ }^{\circ} \mathrm{C}$ for $45 \mathrm{~s}$, and a final extension of $10 \mathrm{~min}$ at $72{ }^{\circ} \mathrm{C}$. Amplified products were separated on $2.5 \%$ agarose gels $(1.3 \%$ agarose gel for the house keeping gene $18 \mathrm{~s}$ rRNA) and visualized with SybrSafe.

\section{In vivo xenograft experiments}

Animal experiments were approved by the Ethical Committee of Institut Gustave Roussy (IGR, Villejuif, France, Ref: 5791/2016062208393968) (CEEA IRCIV/IGR n²6, French Ministry of Research). Female nu/nu mice were obtained from the Centre d'élevage Janvier and used at 6 weeks of age. Five million human osteosarcoma U2OS wild type (WT), U2OS stably expressing XBP1 $\triangle \mathrm{DBD}$ venus or U2OS stably expressing pSMALB-ATF4.5rep cells were diluted in $100 \mu \mathrm{L}$ Dulbecco's modified Eagle medium (DMEM) subsequently subcutaneously injected in both flanks respectively in three groups of 21 mice. After 6-8 weeks, when tumors became palpable, each group was treated with $1 \mathrm{mg} / \mathrm{kg}$ tunicamycin, $5.17 \mathrm{mg} / \mathrm{kg}$ mitoxantrone or DMEM by intratumoral injection. Tumors were resected after $6 \mathrm{~h}$ of treatment from the WT group, after $12 \mathrm{~h}$ from ATF4 and XBP1s groups and after $48 \mathrm{~h}$ from each group treated with mitoxantrone. Tumors were immediately fixed in $3.7 \%$ paraformaldehyde (PFA, F8775 Sigma Aldrich) overnight and then incubated in sucrose gradient. Tumors were sectioned into $5-\mu \mathrm{M}$ slices using a cryostat microtome. Immunostaining was performed after washing and blocking free aldehyde groups with $100 \mathrm{mM}$ glycine for $5 \mathrm{~min}$ followed by $50 \mathrm{mM} \mathrm{NH}_{4} \mathrm{Cl}$ for $20 \mathrm{~min}$, respectively.

\section{Intracellular immunofluorescence staining and fluorescence microscopic analysis}

Cells were seeded in 384-well plates (Greiner Bio-one; Kremsmünster, Austria). After treatment, cells were washed with phosphate-buffered saline (PBS) and fixed in PFA $4 \%$ containing $4 \mu \mathrm{M}$ Hoechst 33342 for $20 \mathrm{~min}$ at room temperature. Then, cells were washed again with PBS and unspecific antibody binding was reduced with blocking buffer (2\% BSA in PBS) for $30 \mathrm{~min}$. After permeabilization with $0.1 \%$ Triton X-100 (Sigma-Aldrich) for $5 \mathrm{~min}$, cells were incubated with primary antibody diluted in blocking buffer overnight. The following day, cells were washed three times with PBS and incubated with Alexafluor ${ }^{\circledR}$ coupled secondary antibody (Thermo Fisher scientific) for 45 min. Finally, cells were washed three times with PBS and fluorescent microscopic analyses were performed by means of an ImageXpress Micro XL automated microscopes (Molecular Devices, Sunnyvale, CA, USA), 
equipped with a $20 \times$ PlanApo objective. Acquired images were segmented into regions of interest (ROI) using the MetaXpress software (molecular devices). Parameters including fluorescence intensity, ROI dimensions, ROI number were extracted and further normalized, statistically evaluated and graphically depicted using the freely available software R (https://www.r-project.org). Intrinsic fluorescence emission of some colored drugs has been measured in U2OS WT cells and was subtracted from biosensor assessments in order to avoid false positive results.

\section{Surface-immunofluorescence staining and fluorescence microscopic analysis}

Cells were seeded in 384-well plates (Greiner Bio-one; Kremsmünster, Austria). After treatment, cells were kept on ice and washed twice with cold PBS. Then primary antibody, diluted in blocking buffer (2\% BSA in PBS), was added for $30 \mathrm{~min}$. After two washes in PBS, cells were incubated with Alexafluor ${ }^{\circledR}$-coupled secondary antibody (Thermo Fisher scientific) and $4 \mu \mathrm{M}$ Hoechst 33342 diluted in $2 \%$ BSA for $30 \mathrm{~min}$. Then cells were washed once with cold PBS. Finally, cells were incubated for $10 \mathrm{~min}$ with Wheat Germ Agglutinin, Alexa Fluor ${ }^{\circledR} 647$ conjugate (Invitrogen), washed once again with cold PBS and fluorescence microscopic analyses were directly performed.

\section{ATP assays}

Cells were seeded in 384-well plates (Greiner Bio-one; Kremsmünster, Austria). After treatment, cells were labeled with $5 \mu \mathrm{M}$ quinacrine in Krebs-Ringer solution $(125 \mathrm{mM}$ $\mathrm{NaCl}, 5 \mathrm{mM} \mathrm{KCl}, 1 \mathrm{mM} \mathrm{MgSO}$, $0.7 \mathrm{mM} \mathrm{KH}_{2} \mathrm{PO}_{4}, 2 \mathrm{mM}$ $\mathrm{CaCL}_{2}, 6 \mathrm{mM}$ glucose and $25 \mathrm{mM}$ HEPES, $\mathrm{pH}$ 7.4) and 4 $\mu \mathrm{M}$ Hoechst 33342 for $30 \mathrm{~min}$ at $37^{\circ} \mathrm{C}$. Then cells were rinsed in Krebs-Ringer solution and microscopic analyses were performed.

\section{Flow cytometry analysis}

Four thousand U2OS WT cells were seeded in 96-well plates. After $6 \mathrm{~h}$ of treatment, cells were recovered, centrifuged and ecto-CALR was detected by immunofluorescence staining. Cells were treated with mitoxantrone and oxaliplatin for $6 \mathrm{~h}$ and recovered after overnight. In short, cells were incubated for $30 \mathrm{~min}$ at $4^{\circ} \mathrm{C}$ with primary rabbit monoclonal antibody against CALR (\#ab2907, Abcam), then washed and further incubated with secondary Alexafluor $^{\circledR} 488$ goat anti-rabbit IgGs (\#A11034, Thermo Fisher Scientific) for $30 \mathrm{~min}$ at $4{ }^{\circ} \mathrm{C}$. Finally, 4', 6diamidino-2 phenylindole dihydrochloride (DAPI, \# D1306, Thermo Fisher Scientific) was added before the analysis. Samples were analyzed using a CyAn ADP cytofluorometer (Beckman Coulter, Brea, CA, USA) coupled to a HyperCyt loader (Intellicyt, Albuquerque, NM, USA).

\section{Global protein translation}

Ten thousand U2OS cells per well were seeded in 96-well plates. Cells were pretreated for $2 \mathrm{~h}$ with mitoxantrone, oxaliplatin, cycloheximide, tunicamycin, thapsigargin or cisplatin. Then they were treated for $6 \mathrm{~h}$ with the same drugs associated with Click-iT ${ }^{\circledR}$ AHA (L-azidohomoalanine) reagent from Invitrogen (C10289) diluted in DMEM supplemented with dialyzed FBS without methionine. Cells were washed once with PBS and fixed in 4\% PFA containing $4 \mu \mathrm{M}$ Hoechst 33342 for $20 \mathrm{~min}$ at room temperature. After two washes with $3 \%$ BSA in PBS, $0.1 \%$ Triton $\mathrm{X}-100$ was added for $20 \mathrm{~min}$ at room temperature. Cells were washed twice with 3\% BSA in PBS and incubated in

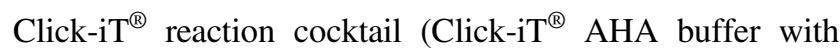
Click-iT $^{\circledR}$ supermix containing Alexa Fluor ${ }^{\circledR} 488$ alkyne) for $30 \mathrm{~min}$. After three washes with $3 \%$ BSA in PBS, fluorescent microscopic analyses were directly performed.

\section{Hyperploid cell lines}

Two hyperploid clones containing 8n DNA for the cell lines EL4 (T1, T2) and HCT116 (T1,T2) were obtained after treatment of parental diploid EL4 and HCT116 cells with $100 \mathrm{nM}$ nocodazole for $48 \mathrm{~h}$. Cells were washed and cultured for 15 days under standard culture conditions followed by FACS-assisted cloning.

\section{Transcriptome analysis}

$5.10^{5}$ EL4T1 or EL4T2 hyperploid clones or parental EL4 cells were injected into either immunocompetent C57B1/6 mice or immunodeficient NOD/SCID mice purchased from Harlan France Sarl (Gannat, France) and The Jackson Laboratory (Bar Harbor, ME, USA), respectively. Animal experiments were approved by the Ethical Committee of IGR (Villejuif, France, Ref: 2016_062_ex047_5251) (CEEA IRCIV/IGR n²6, French Ministry of Research). Hyperploid or parental tumors were resected, recovered and dispersed with $0.25 \%$ trypsin-EDTA for $30 \mathrm{~min}$ at $37^{\circ} \mathrm{C}$, followed by mechanical dispersion and cell culture. RNA was then extracted following the manufacture's instructions of the NucleoSpin(R) RNA/Protein Kit (MACHEREYNAGEL). Five samples issued from different mice from EL4T1 and EL4T2 derived tumors, as well as samples issued from the EL4T1 and EL4T2 hyperploid clones or EL4 parental cell line, were given to the genomic platform of Gustave Roussy Institute, in the context of the Taxe d'Apprentissage TA PAGA_24 2013 to perform a whole 
genome expression array. Upstream Regulator Analysis (IPA ${ }^{\circledR}$, QIAGEN Redwood City, http://www.qiagen.com/ ingenuity) was used to compute transcription factor activities, using log-fold change of differential expression (hyperploid cells compared to parental cells) and (immunodeficient mice compared to immunocompetent mice) as transcription factor target expression. A list of transcription factors were obtained from the IPA ${ }^{\circledR}$ software database. ATF4 target genes were also obtained from the IPA ${ }^{\circledR}$ software database.

\section{Construction of the mathematical predictor of ICD}

The model was built based on a training data set composed of 75 drugs. Each drug was associated with (1) a set of 11 biological scores and (2) a set of 319 descriptors (calculated using the Chemistry Development Kit via the rcdk package available on CRAN) plus 24 (imported from Pubchem database). First, irrelevant descriptors were removed by calculating the median absolute deviation and discarding those with a value lower than $10^{-4}$. The final set of 93 descriptors was centered and scaled to unit variance, subjected to principal component analysis (PCA) to derive up to 11 uncorrelated linear combinations, comprising up to $94 \%$ of total variance. Since we had no expectation about the relative contribution of each individual ICD component to the total ICD 'response', we simply summed up the biological scores (in their $z$-score scale, see above) and looked for optimal models that can best describe each biological score combination to the PCA-reduced descriptor matrix by least-square regression. Furthermore, we imposed two additional constrains to select our final predictive model: (1) the predicted score of six prototypical ICD inducers (daunorubicin, docetaxel, doxorubicin, mitoxantrone, oxaliplatin, paclitaxel) must be in the top 10 (amongst the 75 drugs of the training set), (2) largest spread in the predicted score. The final model is made of an ICD score and the 11 dimensions. We then applied this model onto two validation data sets for which data transformation used the same scaling/rotation parameters as calculated for the training set. We predicted the ICD score from our final regression model and compared the distribution of known inducers (not used for model construction) among putative hits with the distribution of remaining drugs, by means of the Kolmogorov-Smirnov test.

\section{Statistical analyses}

Data are presented as mean \pm SD (or SEM if noticed). All statistical analyses were performed using $\mathrm{R}$ software. Student's $t$-test was used to compare parametric data. Correlation was performed using a Pearson test. Matrix correlation has been calculated with raw data expressed as $z$-scores. Heatmaps (Figs. 5a and 6h) have been realized after sigmoidal scaling of raw data. For all tests, the statistical significance level was $* p<0.05, * * p<0.01$, $* * * p<0.001$.

Acknowledgements We thank David Enot for his help in mathematical modeling and Gautier Stoll for his help in statistical analysis in hyperploid experiments, Prof. Dr. Claudio Hetz for help with the XPB1 reverse transcription PCR, Prof. Dr. John E. Dick for sharing the pSMALB-ATF4.5rep plasmid [52] and Dr. Silke Nock and Prof. Dr. Peter Walter for the U2OS GFP-ATF6 cell line. GK is supported by the Ligue contre le Cancer (équipe labelisée); Agence National de la Recherche (ANR) - Projets blancs; ANR under the frame of E-Rare2, the ERA-Net for Research on Rare Diseases; Association pour la recherche sur le cancer (ARC); Cancéropôle Ile-de-France; Institut National du Cancer (INCa); Institut Universitaire de France; Fondation pour la Recherche Médicale (FDM20140630126 and FDM 40739); the European Commission (ArtForce); the European Research Council (ERC); the LeDucq Foundation; the LabEx Immuno-Oncology; the RHU Torino Lumière, the SIRIC Stratified Oncology Cell DNA Repair and Tumor Immune Elimination (SOCRATE); the SIRIC Cancer Research and Personalized Medicine (CARPEM); and the Paris Alliance of Cancer Research Institutes (PACRI). PL is supported by the Chinese Scholarship Council and LZ by la Ligue contre le cancer. LS is supported by Fondation ARC projects. JH owns a Fondation Philantropia's fellowship. LG is supported by Fundação para a Ciência e a Tecnologia (FCT).

\section{Compliance with ethical standards}

Conflict of interest The authors declare that they have no conflict of interest.

\section{References}

1. Sharma P, Allison JP. Immune checkpoint targeting in cancer therapy: toward combination strategies with curative potential. Cell. 2015;161:205-14.

2. Palucka AK, Coussens LM. The basis of oncoimmunology. Cell. 2016;164:1233-47.

3. Pitt JM, Vetizou M, Daillere R, Roberti MP, Yamazaki T, Routy $\mathrm{B}$, et al. Resistance mechanisms to immune-checkpoint blockade in cancer: tumor-intrinsic and -extrinsic factors. Immunity. 2016;44:1255-69.

4. Sharma P, Hu-Lieskovan S, Wargo JA, Ribas A. Primary, adaptive, and acquired resistance to cancer immunotherapy. Cell. 2017;168:707-23.

5. Topalian SL, Hodi FS, Brahmer JR, Gettinger SN, Smith DC, McDermott DF, et al. Safety, activity, and immune correlates of anti-PD-1 antibody in cancer. N Engl J Med. 2012;366:2443-54.

6. Brahmer JR, Tykodi SS, Chow LQ, Hwu WJ, Topalian SL, Hwu $\mathrm{P}$, et al. Safety and activity of anti-PD-L1 antibody in patients with advanced cancer. N Engl J Med. 2012;366:2455-65.

7. Krysko DV, Garg AD, Kaczmarek A, Krysko O, Agostinis P, Vandenabeele P, Immunogenic cell death and DAMPs in cancer therapy. Nat Rev Cancer. 2012;12:860-75.

8. Kroemer G, Galluzzi L, Kepp O, Zitvogel L. Immunogenic cell death in cancer therapy. Annu Rev Immunol. 2013;31:51-72.

9. Galluzzi L, Buque A, Kepp O, Zitvogel L, Kroemer G. Immunogenic cell death in cancer and infectious disease. Nat Rev Immunol. 2017;17:97-111. 
10. Obeid M, Tesniere A, Ghiringhelli F, Fimia GM, Apetoh L, Perfettini JL, et al. Calreticulin exposure dictates the immunogenicity of cancer cell death. Nat Med. 2007;13:54-61.

11. Michaud M, Martins I, Sukkurwala AQ, Adjemian S, Ma Y, Pellegatti $\mathrm{P}$, et al. Autophagy-dependent anticancer immune responses induced by chemotherapeutic agents in mice. Science. 2011;334:1573-7.

12. Martins I, Wang Y, Michaud M, Ma Y, Sukkurwala AQ, Shen S, et al. Molecular mechanisms of ATP secretion during immunogenic cell death. Cell Death Differ. 2014;21:79-91.

13. Ma Y, Adjemian S, Mattarollo SR, Yamazaki T, Aymeric L, Yang $\mathrm{H}$, et al. Anticancer chemotherapy-induced intratumoral recruitment and differentiation of antigen-presenting cells. Immunity. 2013;38:729-41.

14. Ko A, Kanehisa A, Martins I, Senovilla L, Chargari C, Dugue D, et al. Autophagy inhibition radiosensitizes in vitro, yet reduces radioresponses in vivo due to deficient immunogenic signalling. Cell death Differ. 2014;21:92-9.

15. Ma Y, Galluzzi L, Zitvogel L, Kroemer G, Autophagy and cellular immune responses. Immunity. 2013;39:211-27.

16. Senovilla L, Vitale I, Martins I, Tailler M, Pailleret C, Michaud $\mathrm{M}$, et al. An immunosurveillance mechanism controls cancer cell ploidy. Science. 2012;337:1678-84.

17. Vacchelli E, Ma Y, Baracco EE, Sistigu A, Enot DP, Pietrocola F, et al. Chemotherapy-induced antitumor immunity requires formyl peptide receptor 1 . Science. 2015;350:972-8.

18. Galluzzi L, Buque A, Kepp O, Zitvogel L, Kroemer G. Immunological effects of conventional chemotherapy and targeted anticancer agents. Cancer Cell. 2015;28:690-714.

19. Pietrocola F, Pol J, Vacchelli E, Rao S, Enot DP, Baracco EE, et al. Caloric restriction mimetics enhance anticancer immunosurveillance. Cancer Cell. 2016;30:147-60.

20. Casares N, Pequignot MO, Tesniere A, Ghiringhelli F, Roux S, Chaput N, et al. Caspase-dependent immunogenicity of doxorubicin-induced tumor cell death. J Exp Med. 2005; 202:1691-701.

21. Pfirschke C, Engblom C, Rickelt S, Cortez-Retamozo V, Garris C, Pucci F, et al. Immunogenic chemotherapy sensitizes tumors to checkpoint blockade therapy. Immunity. 2016; 44:343-54.

22. Fridman WH, Pages F, Sautes-Fridman C, Galon J. The immune contexture in human tumours: impact on clinical outcome. Nat Rev Cancer. 2012;12:298-306.

23. Mlecnik B, Bindea G, Angell HK, Maby P, Angelova M, Tougeron $\mathrm{D}$, et al. Integrative analyses of colorectal cancer show immunoscore is a stronger predictor of patient survival than microsatellite instability. Immunity. 2016;44:698-711.

24. Apetoh L, Ghiringhelli F, Tesniere A, Obeid M, Ortiz C, Criollo A, et al. Toll-like receptor 4-dependent contribution of the immune system to anticancer chemotherapy and radiotherapy. Nat Med. 2007;13:1050-9.

25. Vacchelli E, Enot DP, Pietrocola F, Zitvogel L, Kroemer G. Impact of pattern recognition receptors on the prognosis of breast cancer patients undergoing adjuvant chemotherapy. Cancer Res. 2016;76:3122-6.

26. Tesniere A, Schlemmer F, Boige V, Kepp O, Martins I, Ghiringhelli $\mathrm{F}$, et al. Immunogenic death of colon cancer cells treated with oxaliplatin. Oncogene. 2010;29:482-91.

27. Kepp O, Galluzzi L, Lipinski M, Yuan J, Kroemer G. Cell death assays for drug discovery. Nat Rev Drug Discov. 2011;10:221-37.

28. Menger L, Vacchelli E, Adjemian S, Martins I, Ma Y, Shen S, et al. Cardiac glycosides exert anticancer effects by inducing immunogenic cell death. Sci Transl Med. 2012;4:143ra99

29. Sukkurwala AQ, Adjemian S, Senovilla L, Michaud M, Spaggiari S, Vacchelli E, et al. Screening of novel immunogenic cell death inducers within the NCI mechanistic diversity set. Oncoimmunology. 2014;3:e28473

30. Fucikova J, Becht E, Iribarren K, Goc J, Remark R, Damotte D, et al. Calreticulin expression in human non-small cell lung cancers correlates with increased accumulation of antitumor immune cells and favorable prognosis. Cancer Res. 2016;76:1746-56.

31. Fucikova J, Truxova I, Hensler M, Becht E, Kasikova L, Moserova I, et al. Calreticulin exposure by malignant blasts correlates with robust anticancer immunity and improved clinical outcome in AML patients. Blood. 2016;128:3113-24.

32. Donnelly N, Gorman AM, Gupta S, Samali A. The eIF2alpha kinases: their structures and functions. Cell Mol Life Sci. 2013;70:3493-511.

33. Choy MS, Yusoff P, Lee IC, Newton JC, Goh CW, Page R, et al. Structural and Functional Analysis of the GADD34:PP1 eIF2alpha Phosphatase. Cell Rep. 2015;11:1885-91.

34. Harding HP, Zhang Y, Zeng H, Novoa I, Lu PD, Calfon M, et al. An integrated stress response regulates amino acid metabolism and resistance to oxidative stress. Mol Cell. 2003;11:619-33.

35. Lu PD, Jousse C, Marciniak SJ, Zhang Y, Novoa I, Scheuner D, et al. Cytoprotection by pre-emptive conditional phosphorylation of translation initiation factor 2. EMBO J. 2004;23:169-79.

36. Pakos-Zebrucka K, Koryga I, Mnich K, Ljujic M, Samali A, Gorman AM. The integrated stress response. EMBO Rep. 2016;17:1374-95.

37. Wang M, Kaufman RJ. The impact of the endoplasmic reticulum protein-folding environment on cancer development. Nat Rev Cancer. 2014;14:581-97.

38. Bettigole SE, Glimcher LH. Endoplasmic reticulum stress in immunity. Annu Rev Immunol. 2015;33:107-38.

39. Panaretakis T, Kepp O, Brockmeier U, Tesniere A, Bjorklund AC, Chapman DC, et al. Mechanisms of pre-apoptotic calreticulin exposure in immunogenic cell death. EMBO J. 2009;28: 578-90.

40. Panas MD, Ivanov P, Anderson P. Mechanistic insights into mammalian stress granule dynamics. J Cell Biol. 2016; 215:313-23.

41. Talloczy Z, Jiang W, Virgin HWt, Leib DA, Scheuner D, Kaufman RJ, et al. Regulation of starvation- and virus-induced autophagy by the eIF2alpha kinase signaling pathway. Proc Natl Acad Sci USA. 2002;99:190-5.

42. Shen S, Niso-Santano M, Adjemian S, Takehara T, Malik SA, Minoux H, et al. Cytoplasmic STAT3 represses autophagy by inhibiting PKR activity. Mol Cell. 2012;48:667-80.

43. Leavitt R, Schlesinger S, Kornfeld S. Tunicamycin inhibits glycosylation and multiplication of Sindbis and vesicular stomatitis viruses. J Virol. 1977;21:375-85.

44. Lytton J, Westlin M, Hanley MR. Thapsigargin inhibits the sarcoplasmic or endoplasmic reticulum Ca-ATPase family of calcium pumps. J Biol Chem. 1991;266:17067-71.

45. Garg AD, Krysko DV, Verfaillie T, Kaczmarek A, Ferreira GB, Marysael $\mathrm{T}$, et al. A novel pathway combining calreticulin exposure and ATP secretion in immunogenic cancer cell death. EMBO J. 2012;31:1062-79.

46. Bloy N, Sauvat A, Chaba K, Buque A, Humeau J, Bravo-San Pedro JM, et al. Morphometric analysis of immunoselection against hyperploid cancer cells. Oncotarget. 2015;6:41204-15.

47. Harding HP, Novoa I, Zhang Y, Zeng H, Wek R, Schapira M, et al. Regulated translation initiation controls stress-induced gene expression in mammalian cells. Mol Cell. 2000;6:1099-108.

48. Hetz C. The unfolded protein response: controlling cell fate decisions under ER stress and beyond. Nat Rev Mol Cell Biol. 2012;13:89-102.

49. Kepp O, Galluzzi L, Giordanetto F, Tesniere A, Vitale I, Martins I, et al. Disruption of the PP1/GADD34 complex induces calreticulin exposure. Cell Cycle. 2009;8:3971-7. 
50. Guo L, Chi Y, Xue J, Ma L, Shao Z, Wu J. Phosphorylated eIF2alpha predicts disease-free survival in triplenegative breast cancer patients. Sci Rep. 2017;7: 44674.

51. Martins I, Kepp O, Schlemmer F, Adjemian S, Tailler M, Shen S, et al. Restoration of the immunogenicity of cisplatin-induced cancer cell death by endoplasmic reticulum stress. Oncogene. 2011;30:1147-58.
52. van Galen P, Kreso A, Mbong N, Kent DG, Fitzmaurice T, Chambers JE, et al. The unfolded protein response governs integrity of the haematopoietic stem-cell pool during stress. Nature. 2014;510:268-72.

53. Scheuner D, Song B, McEwen E, Liu C, Laybutt R, Gillespie P, et al. Translational control is required for the unfolded protein response and in vivo glucose homeostasis. Mol Cell. 2001;7:1165-76.

\section{Affiliations}

\section{Lucillia Bezu ${ }^{1,2,3,4,5,6}$ - Allan Sauvat ${ }^{2,3,4,5,6}$ - Juliette Humeau ${ }^{1,2,3,4,5,6}$ - Lígia C. Gomes-da-Silva 2,3,4,5,6,7 .

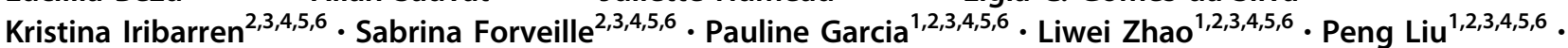 Laurence Zitvogel ${ }^{1,8,9} \cdot$ Laura Senovilla ${ }^{2,3,4,5,6} \cdot$ Oliver Kepp ${ }^{2,3,4,5,6} \cdot$ Guido Kroemer $^{2,3,4,5,6,10,11}$}

1 Faculty of Medicine, University of Paris Sud, KremlinBicêtre, France

2 Metabolomics and Cell Biology Platforms, Gustave Roussy Cancer Campus, Villejuif, France

3 Equipe 11 labellisée Ligue Nationale Contre le Cancer, Centre de Recherche des Cordeliers, Paris, France

4 Institut National de la Santé et de la Recherche Médicale, U1138, Equipe labellisée Ligue Nationale Contre le Cancer, Villejuif, France

5 Université Paris Descartes, Sorbonne Paris Cité, Paris, France

6 Université Pierre et Marie Curie, Paris, France
7 Chemistry Department, University of Coimbra, Coimbra, Portugal

8 Center of Clinical Investigations in Biotherapies of Cancer (CICBT) 1428, Villejuif, France

9 INSERM U1015, Gustave Roussy Cancer Campus, Villejuif, France

10 Pôle de Biologie, Hôpital Européen Georges Pompidou, AP-HP, Paris, France

11 Department of Women's and Children's Health, Karolinska University Hospital, Stockholm, Sweden 